\title{
Bayesian Variable Selection for Logistic
}

\section{Regression}

\author{
Yiqing Tian ${ }^{1}$, Howard D. Bondell ${ }^{1,2}$, Alyson Wilson ${ }^{1}$ \\ North Carolina State University ${ }^{1}$ and University of Melbourne ${ }^{2}$
}

\begin{abstract}
A key issue when using Bayesian variable selection for logistic regression is choosing an appropriate prior distribution. This can be particularly difficult for high-dimensional data where complete separation will naturally occur in the high-dimensional space. We propose the use of the Normal-Gamma prior with recommendations on calibration of the hyperparameters. We couple this choice with the use of joint credible sets to avoid performing a search over the high-dimensional model space. The approach is shown to outperform other methods in high-dimensional settings, especially with highly correlated data. The Bayesian approach allows for a natural specification of the hyperparameters.

Key Words: Joint Credible Region; Normal-Gamma prior; Laplace prior; LASSO.

This is the author manuscript accepted for publication and has undergone full peer review but has not been through the copyediting, typesetting, pagination and proofreading process, which may lead to differences between this version and the Version of Record. Please cite this article as doi: $10.1002 /$ sam.11428
\end{abstract}

This article is protected by copyright. All rights reserved. 


\section{Introduction}

Consider the logistic regression model,

$$
y_{i} \mid \boldsymbol{\beta} \sim \operatorname{Binomial}\left(n_{i}, \pi_{i}(\boldsymbol{\beta})\right), \pi_{i}=\frac{\exp \left(\boldsymbol{x}_{i}^{\prime} \boldsymbol{\beta}\right)}{1+\exp \left(\boldsymbol{x}_{i}^{\prime} \boldsymbol{\beta}\right)}, i=1, \ldots, n
$$

with a data set of $n$ observations and $p$ predictors. The response $y_{i}$ is the number of "successes" observed in $n_{i}$ independent Bernoulli trails with success probability $\pi_{i}$, and $\boldsymbol{x}_{i}=\left(x_{i 0}, x_{i 1}, \ldots, x_{i p}\right)^{\prime}$ is a predictor vector with length $p+1$, where $x_{0}=1$ reflects the inclusion of an intercept. If $n_{i}=1$, all of our data are Bernoulli trials, with either $y_{i}=1$ or $y_{i}=0$. Our problem of interest is variable selection, which means determining which $\beta_{j}=0$ for $j \in\{1, \ldots, p\}$. We note that $\beta_{j}=0$ implies that the $j$ th predictor is excluded from the model. Note that selection is not being performed on the intercept, as we will assume that the intercept should be included in all models. Of particular interest is the problem where $p>n$.

There has been extensive work on variable selection for linear regression models. For example, a variety of methods use stepwise selection coupled with information criteria such as AIC and BIC $[1,19]$. More recently, methods based on penalized likelihood functions have become popular. The key idea of penalization is to impose a constraint on the likelihood function in order to avoid over-fitting. Then $\boldsymbol{\beta}$ is chosen to minimize

$$
l(\boldsymbol{\beta})=\|\boldsymbol{y}-X \boldsymbol{\beta}\|^{2}+\nu P_{\alpha}(\boldsymbol{\beta}),
$$

where $\|\cdot\|$ denotes the Euclidean norm, $P_{\alpha}(\boldsymbol{\beta})$ is a regularization penalty, and $\nu$ is a tuning parameter that is often chosen by cross-validation. With certain choices of regularization, 
some coefficients may collapse to zero, which makes these methods useful for variable selection. Examples include the Dantzig Selector [3], SCAD [6], LASSO [20], group LASSO [21], and Adaptive LASSO [23].

Many of these variable selection methods have been extended to logistic regression models. For example, in forward selection [22], variables are added into the model one-ata-time and the score statistic is used to select the next variable to add. Regularization approaches have also been extended for use with generalized linear models. Friedman et al. [7] developed a cyclical coordinate descent method to fit the generalized linear model and computed a regularization path. Penalties include the $l_{1}$ (LASSO), $l_{2}$ (ridge), and a combination of the two, which is known as the elastic net penalty [24]. Formally, the elastic net penalty is $P_{\alpha}(\boldsymbol{\beta})=(1-\alpha)\|\boldsymbol{\beta}\|_{2}^{2}+\alpha\|\boldsymbol{\beta}\|_{1}$, where $\|\boldsymbol{\beta}\|_{2}^{2}=\sum_{j=1}^{p} \beta_{j}^{2}$ is the ridge penalty and $\|\boldsymbol{\beta}\|_{1}=\sum_{j=1}^{p}\left|\beta_{j}\right|$ is the LASSO penalty. The elastic net can be seen as a compromise between the LASSO $(\alpha=1)$ and ridge penalties $(\alpha=0)$.

Alternatively, Bayesian variable selection methods take a different approach for both linear and logistic regression models. Rather than specifying a single optimal model, they estimate the posterior probability of each possible model. Many Bayesian variable selection methods have been proposed, including Stochastic Search Variable Selection and Gibbs Variable Selection $[4,9]$. Bayes factors are a common metric in Bayesian variable selection $[10,14]$. They measure the evidence from the data in support of the null model. However, a limitation of Bayes factor methods is that they require searching over a $2^{p}$ model space, which is usually not feasible when $p$ is large.

To avoid searching over $2^{p}$ sub-models, George and McCulloch [9] proposed Stochastic Search Variable Selection (SSVS). The basic idea of SSVS is to identify the subsets of 
predictors having the highest posterior probabilities. Latent variables, $\boldsymbol{I}=\left(I_{1}, \ldots, I_{p}\right)$, are introduced into the model to identify subsets: $I_{j}=1$ indicates $x_{j}$ is included in the model. Conditional on the latent variables, $\boldsymbol{I}$, a mixture normal prior is placed on the regression coefficients $\beta_{j}: P\left(\beta_{j} \mid I_{j}\right)=\left(1-I_{j}\right) N\left(0, \sigma_{j}^{2}\right)+I_{j} N\left(0, \tau_{j}^{2}\right)$, with $P\left(I_{j}=1\right)=p_{j}$. The scale, $\tau_{j}^{2}$, is set to a large value so that the non-zero $\beta_{j}$ are included in the final model, and $\sigma_{j}$ is set to a small value so that unimportant $\beta_{j}$ are estimated to be zero. SSVS fits the model using Gibbs sampling to find the posterior distribution on the possible subsets.

Another strategy is to specify a shrinkage prior for the model coefficients. Examples include Normal-Gamma prior, Jeffreys prior, and the Double Exponential prior [11, 13, 15]. As an example, consider the Laplace prior [15], which is a member of the "scale mixture of normals" family. It assumes, conditional on $\tau_{j}$, that $\beta_{j} \sim N\left(0, \tau_{j}\right)$ and $\tau_{j} \sim \operatorname{Exponential}(d)$. The degree of sparsity is tuned by the hyper-parameter $d$.

Since the Laplace prior only involves one parameter, it limits the adaptability of the shrinkage. Griffin and Brown [11] argue that the Laplace prior with a single hyper-parameter fixes the rate of decay of the ordered regression coefficients and leads to unreasonable shrinkage of the parameters. In order to shrink the non-important predictors to zero, one needs to set the mean of the exponential prior to a small value near zero. However, with a fixed decay rate, this may also shrink the true non-zero elements to a value close to zero. More specifically, Griffin and Brown [11] show that if $\zeta_{j}=\tau_{j} / \sum_{j=1}^{p} \tau_{j}$ denotes the proportion of total variability explained by the $j^{\text {th }}$ predictor, and $\zeta_{(j)}$ is the ordered version of $\zeta_{j}$, then the ordered proportion decay rate, $\gamma_{j}=\log \zeta_{(j)}-\log \zeta_{(j+1)}$, does not depend on the hyperparameter $d$. This means that the decay rate of the ordered regression coefficients is fixed. Given this critique, they propose a more generalized form of Laplace prior - the Normal- 
Gamma prior - to overcome the fixed decay rate problem. The Normal-Gamma prior has the form of $\beta_{j} \mid \tau_{j} \sim N\left(0, \tau_{j}\right)$ and $\tau_{j} \sim \operatorname{Gamma}(\lambda, d)$. In this case, the decay of the ordered regression coefficients is determined by the shape parameter $\lambda$, which provides more flexibility on the shrinkage of the parameters.

In this paper, we consider variable selection for logistic regression. Following Bondell and Reich [2], we place a prior on the full set of model parameters and use sparse solutions within posterior credible regions to perform selection. Instead of estimating the posterior probability or the frequency of each possible model, the approach first defines the credible region at a particular coverage level, then searches within the region for the sparsest model. Changing the coverage level yields a sequence of credible sets that can be seen as a sequence of possible models. This paper extends this idea to the logistic regression model, with a focus on the case when $p>n$. Due to the form of the logistic regression model, there is no conjugate prior for the model parameters, but we can still specify priors that have closed-form Gibbs sampling to explore the posterior. To calibrate the hyperparameters, we approximate the distribution of the marginal success probability as a standard Uniform $(0,1)$. Thus we have a marginally non-informative prior, while still maintaining sufficient prior information on the coefficients to perform regularization.

The paper is organized as follows. Section 2 introduces the joint credible region approach and describes the computational issues. Section 3 discusses the use of Laplace and NormalGamma distributions as priors for the logistic regression coefficients and the interpretation of the hyper-parameters. Section 4 details the simulation results, and Section 5 provides conclusions and discussion. 


\section{Joint Credible Region}

The idea behind the penalized credible region approach [2] is to build a sequence of posterior credible regions based on a chosen prior. Given a credible region at a particular level, any point within the region can be considered as a potential value for the parameters. The method chooses the sparsest model represented a point within the region. As the coverage level increases, the model becomes more sparse as the credible region covers more and more space. The expansion of the credible region yields a sequence of credible sets that can be seen as a sequence of models.

Consider the linear regression model, $\boldsymbol{Y}=X \boldsymbol{\beta}+\boldsymbol{\varepsilon}$, with a data set of $n$ observations and $p$ predictors. The errors are assumed to be independently distributed with variance $\sigma^{2}$. The first step for the approach is to build a sequence of posterior credible region by specifying a prior on the full model space. For simplicity, suppose a normal prior is specified for the full model: $\boldsymbol{\beta} \mid \sigma^{2}, \tau \sim N\left(\mathbf{0},\left(\sigma^{2} / \tau\right) I_{p}\right)$. Under this parameterization, $\sigma^{2}$ contributes only to the scale of the posterior, i.e. conditioned on both $\sigma^{2}$ and $\tau$, the posterior is multivariate normal with $\sigma^{2}$ appearing only as a scale factor in the variance, while $\tau$ appears in both the mean and variance. Hence, for any fixed $\tau$, the induced posterior distribution on $\boldsymbol{\beta}$ is elliptical, with the form $P(\boldsymbol{\beta} \mid$ Data $)=H\left[(\boldsymbol{\beta}-\hat{\boldsymbol{\beta}})^{\prime} \Sigma^{-1}(\boldsymbol{\beta}-\hat{\boldsymbol{\beta}})\right]$, where $H$ is a monotone decreasing function, $\hat{\boldsymbol{\beta}}$ and $\Sigma$ are the corresponding posterior mean and covariance matrix. This follows from the fact that the posterior, for fixed $\tau$, after integrating out $\sigma^{2}$ will be a scale mixture of multivariate normals, maintaining the elliptical shape. The form of $H$ is determined by the choice of the prior for $\sigma^{2}$. For example, if the prior for $\sigma^{2}$ is an inverse gamma distribution, then $P(\boldsymbol{\beta} \mid$ Data $)$ is a multivariate t distribution. Note that in practice, 
$\hat{\boldsymbol{\beta}}$ and $\Sigma$ are estimated via MCMC.

If $C_{\alpha}$ denotes a credible region containing $(1-\alpha) \times 100 \%$ of the posterior probability, Bondell and Reich [2] suggest using a elliptical credible region of the form

$$
C_{\alpha}=\left\{\boldsymbol{\beta}:(\boldsymbol{\beta}-\hat{\boldsymbol{\beta}})^{\prime} \Sigma^{-1}(\boldsymbol{\beta}-\hat{\boldsymbol{\beta}}) \leq K_{\alpha}\right\} \text { for some } K_{\alpha}
$$

to represent the proposed posterior credible region at the $\alpha$ level. They also argue that, although placing a prior distribution on $\tau$ no longer maintains the elliptical shape for the posterior distribution, the joint credible region still can be constructed from an elliptical contour having the same form as Equation (3).

Let $\|\boldsymbol{\beta}\|_{0}$ denote the $L_{0}$ norm of vector $\boldsymbol{\beta}$, which is the number of non-zero elements of the vector $\boldsymbol{\beta}$. Consider finding $\tilde{\boldsymbol{\beta}}$ such that

$$
\tilde{\boldsymbol{\beta}}=\operatorname{argmin}\|\boldsymbol{\beta}\|_{0}, \text { subject to } \boldsymbol{\beta} \in C_{\alpha},
$$

which gives a candidate model $A_{n}=\left\{j \mid \tilde{\beta}_{j} \neq 0\right\}$. However, as pointed out in Bondell and Reich [2], using the $L_{0}$ norm, the optimization solution is not unique. Thus, they replace the $L_{0}$ criterion by a combination of the $L_{0}$ and $L_{1}$ criteria. Then by a local linear approximation Zou and Li [25], the optimization problem becomes

$$
\tilde{\boldsymbol{\beta}}=\arg \min \left\{(\boldsymbol{\beta}-\hat{\boldsymbol{\beta}})^{\prime} \Sigma^{-1}(\boldsymbol{\beta}-\hat{\boldsymbol{\beta}})+\lambda_{\alpha} \sum_{j=1}^{p} \frac{\left|\beta_{j}\right|}{\left(\left|\hat{\beta}_{j}\right|\right)^{2}}\right\} .
$$

The proposed solution can be considered as a function of $\lambda_{\alpha}$, where there is a one-toone transformation from $\lambda_{\alpha}$ to $\alpha$. The sequence of solution can be obtained via the LARS algorithm [5] or coordinate optimization [7].

This method is consistent in selection for the fixed-dimensional case, which suggests that the sequence of credible sets will contain the true model with high probability. In addition, 
it avoids an exhaustive search over the $2^{p}$ model space.

Equation (5) has a connection with the Adaptive LASSO [23]. If we adopt a flat or improper prior on $\boldsymbol{\beta}$, with $P(\boldsymbol{\beta}) \propto 1$, then the posterior mean $\hat{\beta}_{j}$ and covariance $\Sigma$ estimates are maximum likelihood estimates. Then the equation is exactly the likelihood function

$(\boldsymbol{\beta}-\hat{\boldsymbol{\beta}})^{\prime} \Sigma^{-1}(\boldsymbol{\beta}-\hat{\boldsymbol{\beta}})$ plus a penalty term $\sum_{j=1}^{p} \frac{\left|\beta_{j}\right|}{\left(\left|\hat{\beta}_{j}\right|\right)^{2}}$, where $w=\frac{1}{\left(\left|\hat{\beta}_{j}\right|\right)^{2}}$ can be viewed as the penalization weight of each regression coefficient. From this point of view, the problem reduces to a Adaptive LASSO problem, with particular weights using the inverse of the square of the initial estimate. However, an improper prior is often inappropriate. Particularly, in the case that $p>n$, an improper prior will result in an improper posterior, as neither the likelihood nor prior would make the model identifiable.

\section{Generalization to Logistic Regression}

We extend the method described in Section 2 to the logistic regression model. We propose to use an elliptical contour for the joint credible region, compute the posterior mean and variance, then find the sparse solution by applying Equation (5). We note that Equation (5) assumes that we use ellipsoidal credible regions centered at the posterior mean and defined via the posterior covariance matrix. For logistic regression, the posterior distribution will be non-elliptical due to the form of the likelihood. However, although they would not be the highest-density posterior regions, ellipsoidal regions of this form are valid credible regions for any distribution, as varying the cutoff can obtain any desired coverage. These would not be the smallest volume confidence regions, but they remain valid, and are those that would be used in practice. Thus we may still use the derivation to Equation (5) as the approach to 
find the sparse solution within these elliptical credible regions. The issue we now consider is the choice of prior distributions for the logistic regression.

\subsection{Choice of Prior}

The choice of the prior distribution for the parameters of the logistic regression is key for Bayesian inference. A widely used class of priors is the family of scale mixture of normals priors. We consider a common member of the family, the Normal-Gamma prior, and a special case of Normal-Gamma prior, the Laplace prior. By varying the choice of hyper-parameters in the Normal-Gamma prior, we can adjust the sparseness of the regression coefficients, which helps achieve the goal of varying shrinkage. Compared with the Laplace prior, the Normal-Gamma prior is more flexible and can achieve better performance.

The most general form of a scale mixture of normals family can be written as

$$
f(\boldsymbol{\beta} \mid \boldsymbol{\mu}, \xi)=\int \phi(\boldsymbol{\beta} \mid \boldsymbol{\mu}, \Sigma) g(\Sigma \mid \xi) d \Sigma
$$

where $\boldsymbol{\beta}$ are the unknown coefficients, $\phi$ denotes the normal density, and $g$ is any proper prior density for the variance matrix $\Sigma$ with hyperparameter $\xi$. Our choice of Normal-Gamma prior arises if $g$ is a gamma distribution.

We use the following hierarchical prior specification for the regression coefficients $\boldsymbol{\beta}$ in Equation (1):

$$
\begin{aligned}
\boldsymbol{\beta} \mid \boldsymbol{\tau} & \sim N(0, B), \\
B & =\operatorname{diag}\left(\tau_{0}, \tau_{1}, \ldots, \tau_{p}\right), \\
\tau_{j} \mid \lambda, d & \sim \operatorname{Gamma}(\lambda, d), j=1, \ldots, p,
\end{aligned}
$$


where $\operatorname{Gamma}(x \mid \lambda, d)$ is the gamma distribution with shape parameter $\lambda>0$ and rate parameter $d>0$ :

$$
\operatorname{Gamma}(x \mid \lambda, d)=\frac{d^{\lambda}}{\Gamma(\lambda)} x^{\lambda-1} \exp \{-d x\}
$$

Equation (7) shows that each regression coefficient $\beta_{j}$ has a normal prior distribution conditional on the scale parameter $\tau_{j}$. For the intercept, we fix its variance parameter at a known constant $\left(\tau_{0}=5\right)$, which is flat for a logistic regression model. For the remaining variance parameters, we specify a gamma distribution in the hierarchy.

\subsection{Choosing Hyper-Parameters}

The choice of hyper-parameters, $\lambda$ and $d$, in the Normal-Gamma prior is important since they play key roles in determining the amount of shrinkage, and thus affect the variable selection results. In this paper, we explicitly tune the hyper-parameters $\lambda$ and $d$ by considering their effects on the marginal prior distribution of $\boldsymbol{\beta}$.

Note that the ratio $\lambda / d$ is the prior mean of $\tau_{j}$, and $\lambda / d^{2}$ is the prior variance of $\tau_{j}$. These choices explicitly affect the marginal distribution of $\beta_{j}$. We want to choose $\lambda$ and $d$ such that non-zero $\beta_{j}$ are significantly different from 0 with high probability, while the "noise" parameters shrink to values close to 0 . Recall that $\tau_{j} \sim \operatorname{Gamma}(\lambda, d)$ and that the prior distribution for $\beta_{j}$ is $N\left(0, \tau_{j}\right)$ conditional on $\tau_{j}$. We want to have large $\tau_{j}$ for non-zero/important coefficients and small values for non-important variables. 


\subsubsection{Calibration of the Prior Mean}

It follows from Equation (1) that the success probability for a subject with covariate vector $\boldsymbol{x}$ is $\pi_{x}=\frac{\exp \left(\boldsymbol{x}^{\prime} \boldsymbol{\beta}\right)}{1+\exp \left(\boldsymbol{x}^{\prime} \boldsymbol{\beta}\right)}$. The prior for $\boldsymbol{\beta}$ and the distribution of $\boldsymbol{x}$ defines a distribution on the overall success probability of the population, $\pi$, which marginalizes out both $\boldsymbol{x}$ and $\boldsymbol{\beta}$, is implied by the prior for $\boldsymbol{\beta}$. There is often some prior knowledge about the success probability of the population. For example, Hanson et al. [12] presents a version of the g-prior for $\boldsymbol{\beta}$ such that the prior distribution on the overall population logistic predictive probabilities of success can be set to match a beta distribution, $\operatorname{Beta}(a, b)$. We adopt the same strategy, and our goal is to model the marginal prior on $\boldsymbol{\beta}$ to induce a distribution on the marginal predictive probability $\pi$ that approximately matches some $\operatorname{Beta}(a, b)$. If no prior information is given on the marginal predictive probability $\pi$, we set $\operatorname{Beta}(1,1)=\operatorname{Uniform}(0,1)$ for $\pi$. In Hanson et al. [12], they demonstrate that setting the variance component, $\tau_{j}$, at $3 / p$ induces an approximately Uniform $(0,1)$ distribution on the predictive probability. In our setting, with a hyper-prior assigned to $\tau_{j}$, the variance component itself is a random quantity. The value $\lambda / d$ determines the mean of this variance component in the prior of $\boldsymbol{\beta}$. We thus set $\mathrm{E}\left(\tau_{j}\right)=\lambda / d=3 / p$ to induce an approximately $\operatorname{Uniform}(0,1)$ distribution on $\pi$. Note that $\tau_{j} \neq 3 / p$, but that it varies around that value.

This can be illustrated by a simple simulation. Figure 1 shows the effect of different choices of $\lambda / d$ on the induced predictive probability distribution. With $n=200, p=200$ and $d=2$, three choices of $\lambda / d$ are considered: $\lambda / d=(0.1,0.015,0.001)$. A vector of coefficients $\boldsymbol{\beta}=\left(\beta_{1}, \ldots, \beta_{p}\right)$ is generated from a multivariate normal distribution $N(\mathbf{0}, \boldsymbol{\tau})$, where the variance components $\boldsymbol{\tau}=\left(\tau_{1}, \ldots, \tau_{p}\right)$ are drawn independently from a $\operatorname{Gamma}(\lambda, d)$. We 
repeat this 10,000 times and compute the predictive probabilities. Figure 1(a) corresponds to $\lambda / d=0.1>3 / p$, where the induced predictive probability has a $U$ shape, while Figure $1(\mathrm{~b})$ shows $\lambda / d=0.001<3 / p$ where the simulated predictive probability has a inverted $U$ shape. Figure $1(\mathrm{c})$ is $\lambda / d=3 / p$, the case that the predictive probability is approximately uniform.

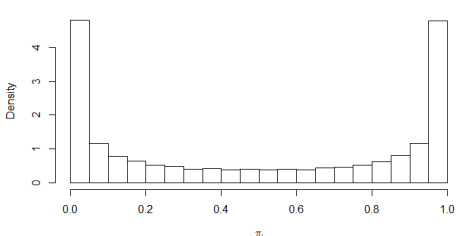

(a) $\lambda / d=0.1$

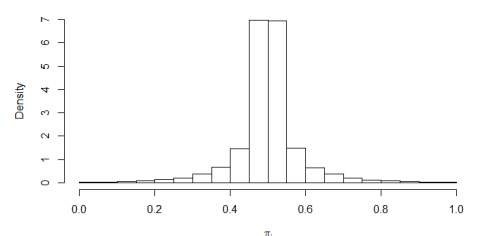

(b) $\lambda / d=0.001$

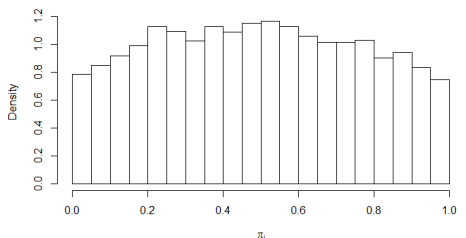

(c) $\lambda / d=0.015$

Figure 1. The induced prior predictive probability distribution $\pi$ based on $n=200, p=200$, $d=2$.

\subsubsection{Calibration of the Prior Variance}

Varying shrinkage of the coefficients is another consideration for tuning. Griffin and Brown [11] discussed the effect of changing the prior variance, $\operatorname{Var}\left(\tau_{j}\right)=\lambda / d^{2}$, on prior beliefs about the proportion of important variables. Let $\varphi$ be the total prior variance of $\boldsymbol{\beta}$ (excluding $\beta_{0}$ ), where $\varphi=\tau_{1}+\ldots+\tau_{p}$. Since each $\tau_{j}$ represents the variation in the $j^{t h}$ predictor, for the proposed model (Equation (7)), a small $\tau_{j}$ is likely to shrink the corresponding regressor $\beta_{j}$ to a value close to 0 , and a large $\tau_{j}$ would suggest a regressor significantly different from 0 . If the total variance $\varphi$ and the prior mean of the variance component $\tau_{j}$ are fixed, then we can adjust each $\tau_{j}$ to control the shrinkage. Note that since the mean is $\lambda / d$ and variance is $\lambda / d^{2}$, the pair $(\lambda / d, d)$ fully specifies the gamma distribution. We have set $\lambda / d=3 / p$ to control the expectation, and now we consider $d$ to control the variance. 
Suppose that we believe the number of important variables is $q$. If $\tau_{(1)}, \ldots, \tau_{(p)}$ are the ordered variance components, then $\delta=\sum_{j=1}^{q} \tau_{(j)} / \sum_{j=1}^{p} \tau_{j}$ can be interpreted as the proportion of the total prior variance in $\boldsymbol{\beta}$ explained by the $q$ largest $\tau_{j}$. Therefore, to control sparseness, we want most of the variation in $\boldsymbol{\beta}$ to be explained by a small proportion of $\tau$. For a fixed $q$, we choose $d$ satisfy the following equation:

$$
\operatorname{mean}\left(\frac{\sum_{j=1}^{q} \tau_{(j)}}{\sum_{j=1}^{p} \tau_{j}}\right) \approx 1-\varepsilon
$$

where $\varepsilon$ is a small prespecified value, and the prior mean of $\tau_{j}$ is fixed at $3 / p$.

This technique can be used to specify both "tight" and "smooth" priors. If the choice of hyper-parameters leads to most of the variance in $\boldsymbol{\beta}$ concentrating on only a few regressors, then we call it a "tight" prior. On the other hand, if the variance in $\boldsymbol{\beta}$ is spread out among more regressors, we consider it a "smooth" prior. Note that, for any $p$ and $\varepsilon$, there is oneto-one correspondence between the degree of sparseness and the value of $\lambda / d^{2}$. Figure 2 plots the prior important variable proportion, $q / p$, against the prior variance, $\lambda / d^{2}$. It shows larger variances associated with smaller values of $q$.

\subsubsection{Laplace Prior}

The Laplace prior is a special case of the Normal-Gamma prior with $\lambda=1$. Since $\lambda$ is fixed, there is less flexibility in specifying the degree of sparseness since the only flexibility is determined by $d$. We choose $d$ to induce an approximately Uniform $(0,1)$ distribution on the predictive probability by setting $1 / d=3 / p$. Then both the prior mean $1 / d$ and prior variance $1 / d^{2}$ are fixed, which induces a prior on the corresponding number of important variables. Our tuning results show that the Laplace prior is automatically a smooth prior 


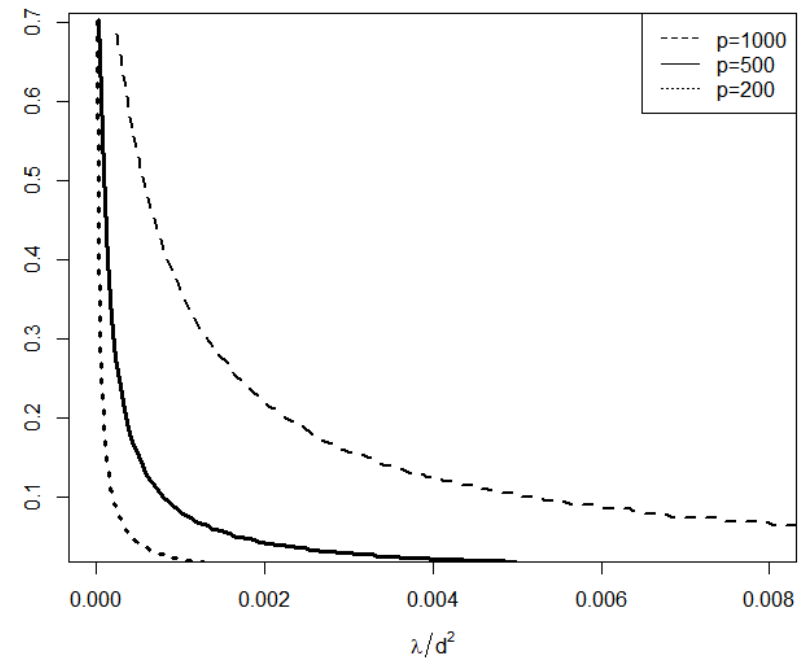

Figure 2. Relationship prior important variable proportion to prior variance.

and applies almost equal shrinkage to all of the coefficients, which means that the number of induced important variables $q$ is close to dimension of the predictors.

\subsubsection{Connection to Spike-and-Slab Priors}

The spike-and-slab prior is widely used in Bayesian variable selection (see for example, Gan et al. [8], George and McCulloch [9], Rockova [17], Rockova and George [18]). The general form of the spike-and-slab prior is

$$
\pi\left(\beta_{i} \mid \gamma_{i}, \lambda_{0}, \lambda_{1}\right)=\left(1-\gamma_{i}\right) \eta_{0}\left(\beta_{i} \mid \lambda_{0}\right)+\gamma_{i} \eta_{1}\left(\beta_{i} \mid \lambda_{1}\right)
$$

where $\gamma_{i} \in\{0,1\}$ is a latent variable indicating whether the variable is included in the model. $\eta_{0}\left(\cdot \mid \lambda_{0}\right)$ is the "spike" distribution that is concentrated near zero with a "small" variance, and $\eta_{1}\left(\cdot \mid \lambda_{1}\right)$ is the "slab" distribution, which is diffuse with a "large" variance. The idea is that if the data suggest that $\gamma_{i}=0$, then $\beta_{i}$ is small enough that it should be excluded from the final model. As examples, George and McCulloch [9] choose normal distributions for $\eta_{0}$ and $\eta_{1}$, and spike-and-lab LASSO [17] chooses $\eta_{0}$ and $\eta_{1}$ to be Laplace distributions. 
We are interested in comparing how the shrinkage of the spike-and-slab LASSO differs from the proposed Normal-Gamma prior. Following the strategy of Griffin and Brown [11], we match the spike-and-slab LASSO with the proposed Normal-Gamma prior by setting the mixing proportion $\gamma_{1}=\gamma_{2}=\ldots=\gamma_{p}=\gamma=q / p$, which is just our prior belief on the proportion of important predictors. Figure 3 shows the shrinkage factor [11] $S(\hat{\beta})$ against $\hat{\beta}$ for a single variable under various values of standard deviation of $\hat{\beta}, S E(\hat{\beta})$, for linear regression model. Since

$$
E(\beta \mid \hat{\beta})=\hat{\beta}(1-S(\hat{\beta}))
$$

large values of $S(\hat{\beta})$ indicates a larger shrinkage. We set $q=9, p=200, \varepsilon=0.05, \lambda_{0}=0.001$, and pick $\lambda_{1}$ to satisfying $\lambda / d=2(1-\gamma) \lambda_{0}^{2}+2 \gamma \lambda_{1}^{2}$ to ensure that $S E(\hat{\beta})$ is the same under the two priors.

Figure 3 shows that the two priors lead to similar shrinkage. When $\hat{\beta}$ is small, the spikeand-slab LASSO matches the Normal-Gamma prior fairly well. As $\hat{\beta}$ gets larger, we can see that the spike-and-slab LASSO actually shrinks slightly more than the Normal-Gamma prior, which results in an increase in bias on the larger coefficients for the spike-and-slab LASSO. As $S E(\hat{\beta})$ gets larger, the difference in shrinkage between the two increases.

\subsection{Inference}

Even using the Normal-Gamma prior, computation is not straightforward. Introducing a latent Pólya-Gamma random variable into the model [16] allows the inference to be directly implemented. With a normal prior specified on the regression coefficients and the latent variable, all updates can be made using Gibbs sampling with closed-form full conditional 


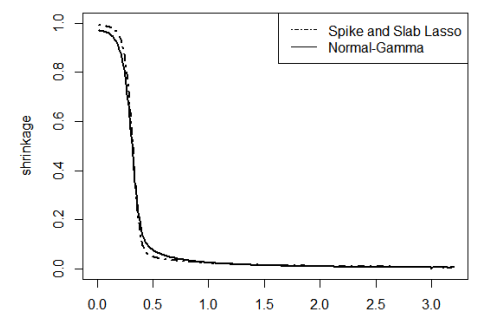

(a) $S E(\hat{\beta})=0.1$

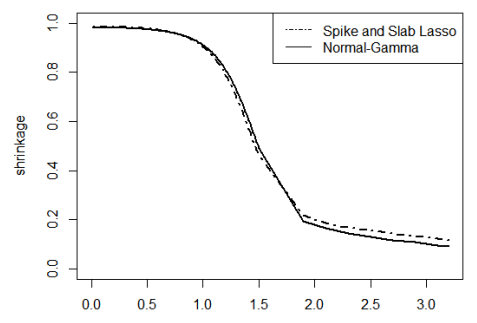

(b) $S E(\hat{\beta})=0.4$

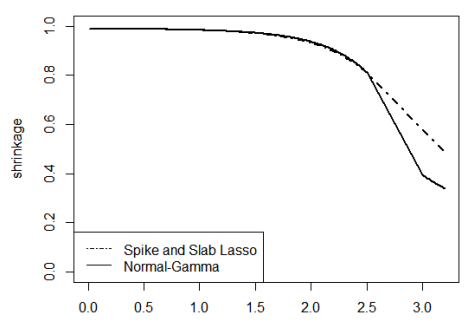

(c) $S E(\hat{\beta})=0.7$

Figure 3. Shrinkage factors for various $S E(\hat{\beta})$ using spike-and-slab LASSO and NormalGamma priors.

distributions.

The induced Pólya-Gamma variable is defined as follows. Let $X \sim P G(b, c)$ represent a Pólya-Gamma random variable. Then for $b>0$ and $c \in \Re$ we have

$$
X \stackrel{D}{=} \frac{1}{2 \pi^{2}} \sum_{k=1}^{\infty} \frac{g_{k}}{(k-1 / 2)^{2}+c^{2} /\left(4 \pi^{2}\right)},
$$

where $g_{k} \sim \operatorname{Gamma}(b, 1)$ are independent gamma random variables. To write the PólyaGamma random variable density function, we have

$$
p(x \mid b, c)=\frac{\exp \left(-\frac{c^{2}}{2} x\right) p(x \mid b, 0)}{\int_{0}^{\infty} \exp \left(-\frac{c^{2}}{2} x\right) p(x \mid b, 0) d x},
$$

where $p(x \mid b, 0)$ is the density of $P G(b, 0)$ random variable.

Consider a Bernoulli trial, $n_{i}=1$, and let $y_{i}$ denote the trial result, with $y_{i} \in\{0,1\}$. Then we have $y_{i} \sim \operatorname{Binomial}\left(1, \pi_{i}(\boldsymbol{\beta})\right)$, where $\pi_{i}=\exp \left(\boldsymbol{x}_{i}^{\prime} \boldsymbol{\beta}\right) /\left\{1+\exp \left(\boldsymbol{x}_{i}^{\prime} \boldsymbol{\beta}\right)\right\}$. To use the Pólya-Gamma strategy, we introduce a sequence of latent Pólya-Gamma random variables $\left\{\omega_{i}\right\}_{i=1}^{n}$ that are generated independently for each observation with distribution

$$
\omega_{i} \sim P G(1,0)
$$


To sample from the posterior distribution, iterate

$$
\begin{array}{r}
\omega_{i} \mid \boldsymbol{\beta} \sim P G\left(1, \boldsymbol{x}_{i}^{\prime} \boldsymbol{\beta}\right), \\
p\left(\boldsymbol{\beta} \mid \boldsymbol{y}, \omega_{1}, \ldots, \omega_{n}, \boldsymbol{\tau}\right) \sim N(m, V), \\
p\left(\tau_{j} \mid \beta_{j}\right) \sim \operatorname{GIG}\left(\lambda-\frac{1}{2}, 2 d, \beta_{j}^{2}\right)
\end{array}
$$

where $\operatorname{GIG}(p, a, b)$ denotes the Generalized Inverse Gamma distribution with parameters $p$, $a$, and $b$ with density

$$
f(x \mid p, a, b)=\frac{(a / b)^{p / 2}}{2 K_{p}(\sqrt{a b})} x^{p-1} \exp \left\{-\frac{1}{2}(a x+b / x)\right\}, x>0
$$

and

$$
\begin{aligned}
& V=\left(B^{-1}+X^{\prime} \Omega X\right)^{-1} \\
& m=V\left(B^{-1} b+X^{\prime} \Omega z\right)
\end{aligned}
$$

with $z=\left(k_{1} / \omega_{1}, \ldots, k_{N} / \omega_{N}\right), k_{i}=y_{i}-n_{i} / 2$, and $\Omega=\operatorname{diag}\left(\omega_{1}, \ldots, \omega_{N}\right)$.

Because it has two free parameters, the Normal-Gamma prior is more flexible in determining the sparseness of the regression coefficients than the Laplace prior. However, for comparison, we also consider using a Laplace prior for the regression coefficients. For the Laplace prior, the Gibbs updates for $\boldsymbol{\omega}$ and $\boldsymbol{\beta}$ are the same as those for the Normal-Gamma prior. The updates for $\tau_{j}$ are simplified by sampling $1 / \tau_{j}$ conditional on $\beta_{j}$ from an inverseGaussian distribution,

$$
\frac{1}{\tau_{j}} \mid \beta_{j} \sim \operatorname{InvGaussian}\left(\sqrt{\frac{2 d}{\left(\beta_{j}\right)^{2}}}, 2 d\right)
$$

If $\mu=\sqrt{\frac{2 d}{\left(\beta_{j}\right)^{2}}}$ and $\nu=2 d$, then the inverse-Gaussian distribution can be written as

$$
f(x \mid \mu, \nu)=\left\{\frac{\nu}{2 \pi x^{3}}\right\}^{1 / 2} \exp \left\{-\frac{\nu(x-\mu)^{2}}{2 \mu^{2} x}\right\} .
$$




\section{Simulation Results}

\subsection{Methods and Metrics}

We use several simulation studies to examine the performance of the joint credible region approach and compare it with other variable selection methods: LASSO, forward selection, and screening. We also examine the impact of hyper-parameter selection for the performance of the joint credible region approach.

With LASSO, we use a $L_{1}$ norm penalized function, $\|\boldsymbol{\beta}\|_{1}$, to obtain the solution path. We implement LASSO using the R package glmnet. Forward selection uses the score test to perform variable selection. More specifically, in the first step, it chooses the variable with largest univariate score statistic. The next step is to find the variable that gives the largest score assuming the variable chosen in the previous step stays in the model. The process is repeated until all variables are included in the model. Screening refers to considering each candidate predictor variable separately via its distribution in each of the groups that are defined by the binary response. In this context, we split the data into two groups, based on the value of the binary response. For each continuous predictor, we perform a two-sample t-test and obtain the p-value for the hypothesis test of no difference between the groups. For categorical predictors, instead of t-tests, chi-square tests for equality of the two discrete distributions are used, or Wilcoxon tests for ordered discrete predictors. The predictors are then ordered by the p-values for the test, so that those with smallest p-values are those predictors whose univariate distributions differ the most across the groups defined by the binary response. The joint credible region approach uses both the Normal-Gamma and 
Table 1. Hyper-parameter tuning results. The first two columns are tuning result for the Normal-Gamma prior, while the last column is the result for the Laplace prior. The numbers in the table are the prior variance $\lambda / d^{2}$ corresponding to each $p, q$, while the numbers in parentheses are the prior belief about the number of important variables.

\begin{tabular}{c||c|c|c}
$\lambda / d^{2}(q / p)$ & Normal-Gamma & Normal-Gamma & Laplace \\
\hline$p=200$ & $0.012(9 / 200)$ & $0.002(40 / 200)$ & $0.000225(140 / 200)$ \\
$p=500$ & $0.005(9 / 500)$ & $0.00102(40 / 500)$ & $0.000036(350 / 500)$ \\
$p=1000$ & $0.0025(9 / 1000)$ & $0.00054(40 / 1000)$ & $0.000009(700 / 1000)$
\end{tabular}

Laplace priors. The posterior is computed using 10,000 MCMC iterations following 5000 burn-in samples for 15,000 iterations in total. The ordered solution path is computed via obtaining the solution path to the optimization described in Equation (5).

The hyper-parameters $\lambda$ and $d$ in the Normal-Gamma prior are tuned as discussed in Section 3. We specify $\varepsilon=0.05$ so that $95 \%$ of the variance in $\boldsymbol{\beta}$ is explained by the $q$ largest regressors. Two beliefs on the number of important variables are considered, $q \in\{9,40\}$ where $q=9$ is true number of important variables. The tuning results are shown in Table 1 for $p \in\{200,500,1000\}$. From the table, we can see that a small prior variance $\lambda / d^{2}$ is associated with a large number of important variables $q$.

In order to assess the performance of the approaches, we consider the ordered solution path of predictors. One issue that arises is separation, particularly when the number of predictors is substantially larger than the sample size. Separation is usually associated with categorical data in logistic or probit regression and occurs when only one category's outcome is observed for a region in the predictor space. For example, for a continuous 
predictor $x$, we might always observe $y=1$ when $x \geq 0$. This is more likely to happen in higher dimensions. For LASSO and forward selection, after following the solution sequence and adding some number of variables, perfect separation may occur. Continuing any further along the sequence will lead to no further improvement, and the solution path will terminate. When this happens, we use screening to order the remaining variables. This is similar to the linear regression setting in that the LASSO and forward selection can only select at most $n$ variables since at that point it will reach perfect fit. However, since our proposed approach will always have a regularized solution, in that the sequence of solutions will end at the posterior mean of the full model, which includes regularization, the full solution path up to all $p$ predictors can be obtained. This is equivalent to the elastic net [24] being able to select $p$ predictors, even with a sample of size $n$, due to the $L_{2}$ regularization term there.

To compare performance, we use Receiver Operating Characteristic (ROC) and PrecisionRecall (PRC) curves for the ordered solution paths. Let true positives (TP) denote the predictors that are included in the model correctly, and false positives (FP) denote the predictors that are incorrectly included in the model. True negatives (TN) are the variables that are correctly excluded from model, and false negatives (FN) are the variables that are wrongly omitted from the model. The ROC curve describes the trade off between type I error and power. It plots the false positive rate (1-specificity) on the $\mathrm{x}$-axis and true positive rate (sensitivity) on the y-axis, where sensitivity measures the proportion of positives that are correctly identified and specificity is the proportion of negatives that are correctly identified. The PRC shows the trade off between power and false discovery rate and plots the true positive rate (sensitivity) on the $\mathrm{x}$-axis and precision on $\mathrm{y}$-axis, where precision is defined as the ratio of true positives to the total number declared as positives. 
In the simulation studies, two different data generating mechanisms are considered: prospective and retrospective data generation. We describe these mechanisms in the following sub-sections.

\subsection{Prospective Data Generation}

For prospective data generation, given the true $\boldsymbol{\beta}$, we use $n=200$ and vary the dimension of the predictors $p \in\{200,500,1000\}$. The correlation between predictors, $x_{i, j}$ and $x_{i, k}$ follows an $\operatorname{AR}(1)$ structure, with $\operatorname{Cor}\left(x_{i j}, x_{i k}\right)=\rho^{|j-k|}$. We choose $\rho \in\{0.5,0.8\}$ to represent relatively low and high correlation. We denote the corresponding variance-covariance matrix by $\Sigma_{p \times p}(\rho)$. For each setting, we generate 250 datasets from a logistic regression model. For a given true $\boldsymbol{\beta}$ and $\Sigma_{p \times p}(\rho)$, we generate $\boldsymbol{x}_{i} \sim N\left(\mathbf{0}_{p \times 1}, \Sigma_{p \times p}(\rho)\right)$. Then we have $\pi_{i}=$ $\exp \left(\boldsymbol{x}_{i}^{\prime} \boldsymbol{\beta}\right) /\left\{1+\exp \left(\boldsymbol{x}_{i}^{\prime} \boldsymbol{\beta}\right)\right\}$ and generate $y_{i}$ conditional on $\pi_{i}$ from a Bernoulli distribution with success probability $\pi_{i}(\boldsymbol{\beta})$.

\subsubsection{Spread Signal}

The first case with prospective data generation has the important variables spread out: the important and non-important variables alternate. The important variables occur every eight places starting from the second position with $\beta_{j}=1.8$ for $j \in\{2,10,18,26,34,42,50,58,66\}$. We have 9 important variables in total.

Figure 4 displays the ROC curve and PRC curve for $p=200$ predictors with a spread signal. The upper two plots show $\rho=0.8$ and lower two show $\rho=0.5$. Under high correlation $(\rho=0.8)$, the joint credible region method using the Normal-Gamma prior shows substantial improvement over the other methods. For low correlation, the performance of all methods 
improves and gives similar performance - except for screening, which does not perform well in either case. This is to be expected, since screening does selection based on the marginal correlation between predictors. High correlation between important predictors helps detect other variables while low correlation does the reverse. When signals are spread out, the correlation between important variables is lower under the $\mathrm{AR}(1)$ structure, which affects the performance of screening.

When comparing the choice of priors, both for low and high correlation, the NormalGamma priors specified with $q=9$ perform best, followed by $q=40$. Although the use of the Normal-Gamma prior tuned with $q=40$ does not perform as well as the case of $q=9$, it still outperforms other non-Bayesian methods. The performance of Laplace prior, which suggests more important variables, is not as good as the Normal-Gamma priors with $q=9$ or $q=40$.

Figure 5 gives the results for $p \in\{500,1000\}$ predictors under high correlation $\rho=0.8$. The plot shows results consistent with the $p=200$ case for both $p \in\{500,1000\}$. The joint credible region method demonstrates substantial improvement over the non-Bayesian methods. The prior tuned with $q=9$ performs best, while Laplace prior does poorly.

Since the important predictors/signals are spread out, the use of Normal-Gamma prior with $q=9$, which suggest fewer important predictors, helps to push all effects to the true important predictor among variables that are highly correlated. As the shrinkage is not identical across all predictors, it help to identify the important variables while shrinking the non-important predictors around it to a value close to zero. However, the Laplace prior puts equal shrinkage to both important and non-important predictors, which results in poorer performance. 
We also performed the simulation study for $p \in\{500,1000\}$ in the relatively easier case of $\rho=0.5$ (results not shown). With low correlation, although all methods perform better, the results are similar to those seen with high correlation.

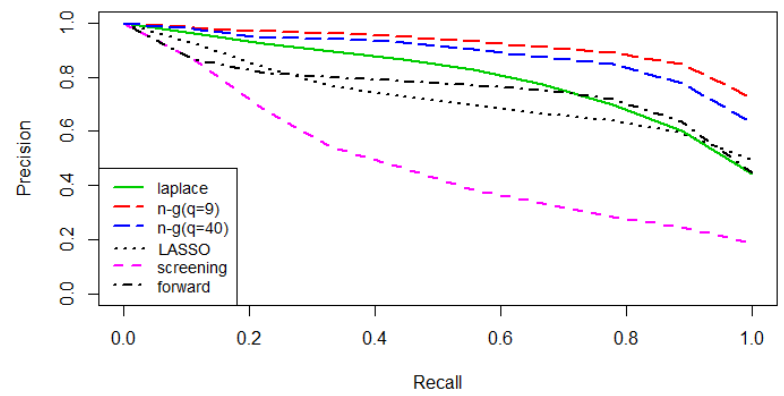

(a) PRC curve, $\rho=0.8$

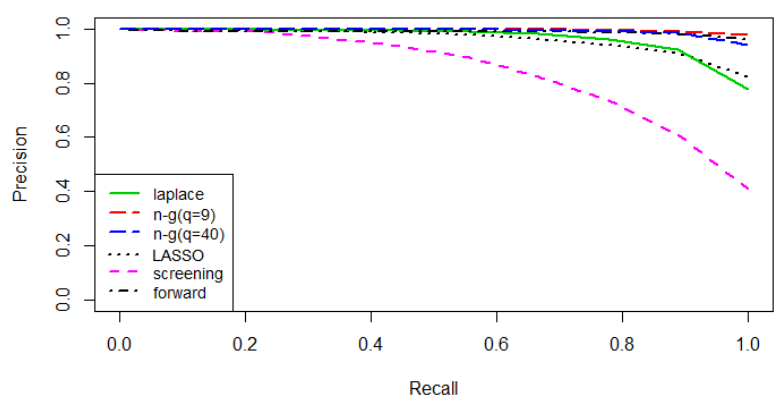

(c) PRC curve, $\rho=0.5$

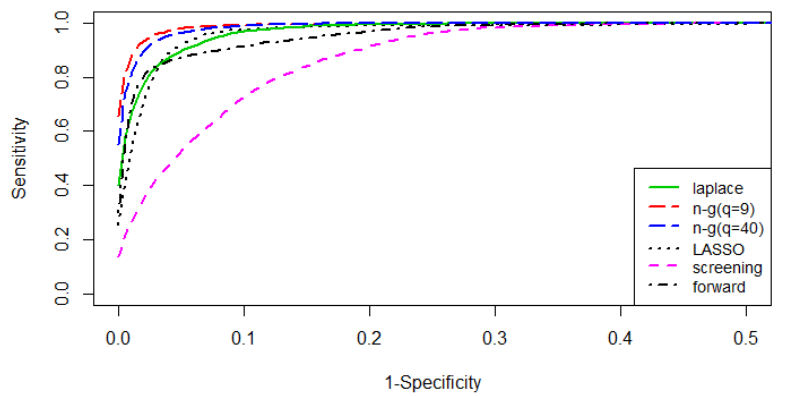

(b) ROC curve, $\rho=0.8$

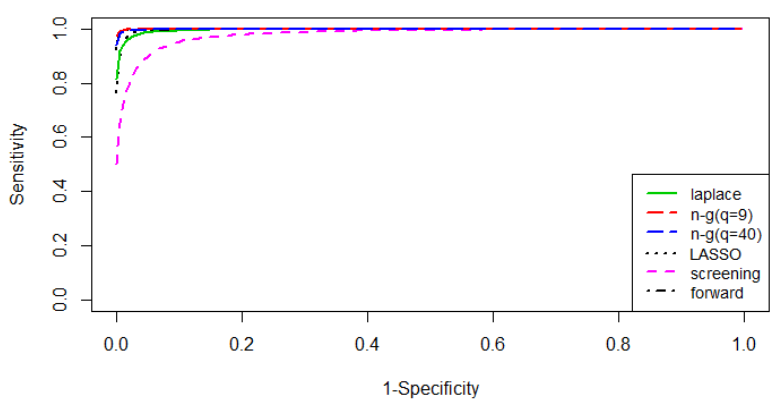

(d) ROC curve, $\rho=0.5$

Figure 4. Mean PRC and ROC curves from prospectively generating 250 datasets for $n=$ $200, p=200, \rho \in\{0.5,0.8\}$ with spread signal.

\subsubsection{Block Signal}

The other case we consider with prospective data generation uses blocks of important predictors. In this setting, we use three blocks with three non-zero coefficients in each. The true 


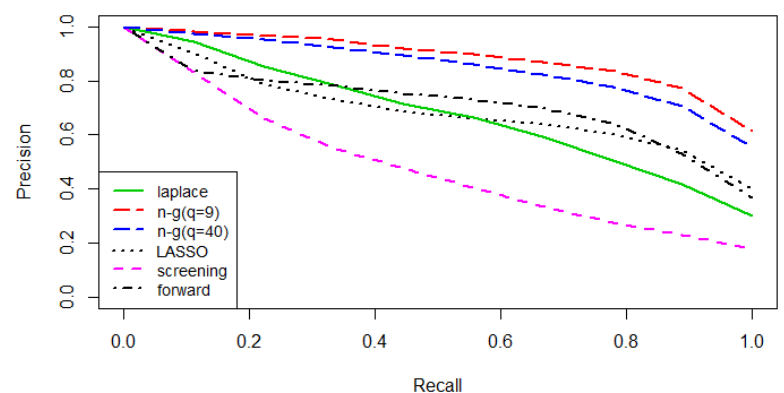

(a) PRC curve, $\rho=0.8, p=500$

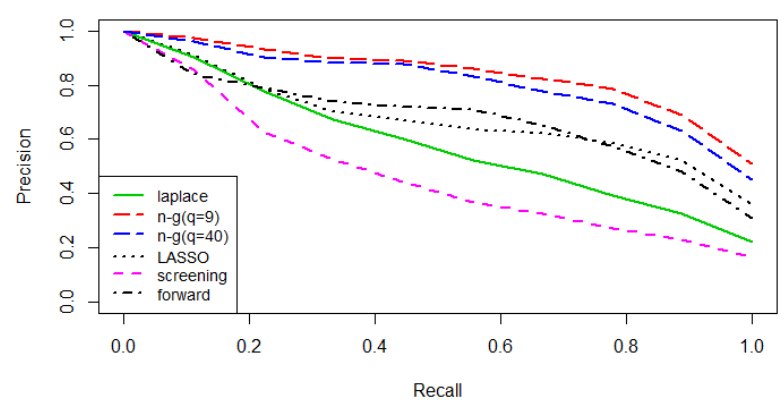

(c) PRC curve, $\rho=0.5, p=1000$

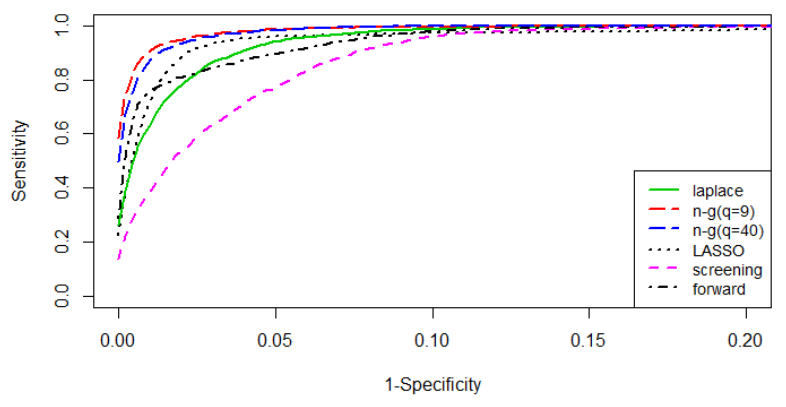

(b) ROC curve, $\rho=0.8, p=500$

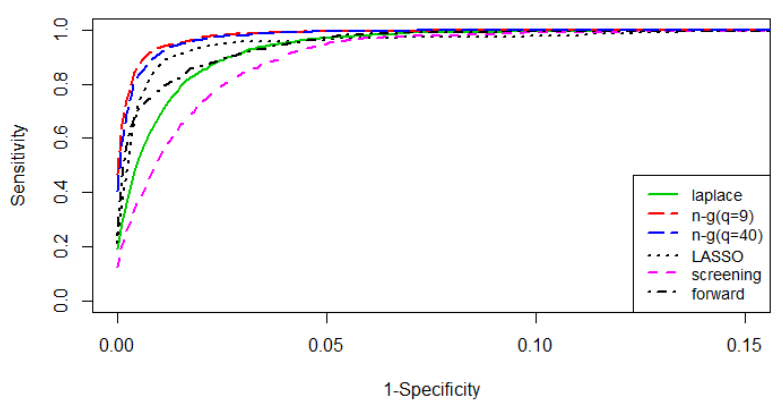

(d) ROC curve, $\rho=0.5, p=1000$

Figure 5. Mean PRC and ROC curve from prospectively generating 250 datasets for $n=200$, $p \in\{500,1000\}, \rho=0.8$ with spread signal.

coefficients are given by $\left(0,0,1.8_{\mathbf{3}}^{\prime}, \mathbf{0}_{18}^{\prime}, 1.8_{\mathbf{3}}^{\prime}, \mathbf{0}_{18}^{\prime}, 1.8_{\mathbf{3}}^{\prime}, \mathbf{0}_{p-46}^{\prime}\right)$, where the subscript denotes the length of the vector. The magnitude of signal we adopt here is same as in the spread signal, with all coefficients positive. We again have 9 important variables.

Figure 6 displays the results in this setting with $p=200$. The upper two plots are for high correlation, while the lower two show results for low correlation. In this case, the Laplace prior gives the best results for both high and low correlation, followed by the Normal-Gamma prior tuned with $q=40$. Forward selection does poorly for each case. 
These results are to be expected. Since important variables are blocked together and highly correlated, the use of the Laplace prior, which is more smooth, gives approximately equal shrinkage to adjacent predictors, avoiding pushing all effects to a single variable. Thus, it can simultaneously pick all the important variables that are correlated with each other. The poor performance of forward selection is perhaps due to the correlation of important predictors, since in each step the method selects variables conditional on the predictors already in the model. It may be hard to select other important variables that highly correlated with the one already chosen.

Figure 7 displays the results for $p \in\{500,1000\}$ with high correlation $(\rho=0.8)$. Compared to the $p=200$ case, we can observe improvement in the performance of the joint credible region method. Again, the Laplace prior outperforms the other methods. Due to the correlation between important predictors, we expect the poor performance of forward selection. We observed consistent results with $\rho=0.5$ for both $p \in\{500,1000\}$.

\subsection{Retrospective Data Generation}

An alternative way of generating data is to generate predictors conditional on the response. This is retrospective, or case control, data generation. Conditional on the response of $y=1$ or $y=0$, we generate two groups of predictors with the same correlation but different mean vectors from multivariate normal distributions. Although the correlation in each group is the same, the marginal distribution of the predictors can be changed according to the magnitude of the shift of the mean vector.

For a given correlation $\rho$, we again use an $\operatorname{AR}(1)$ structure and denote the variance- 


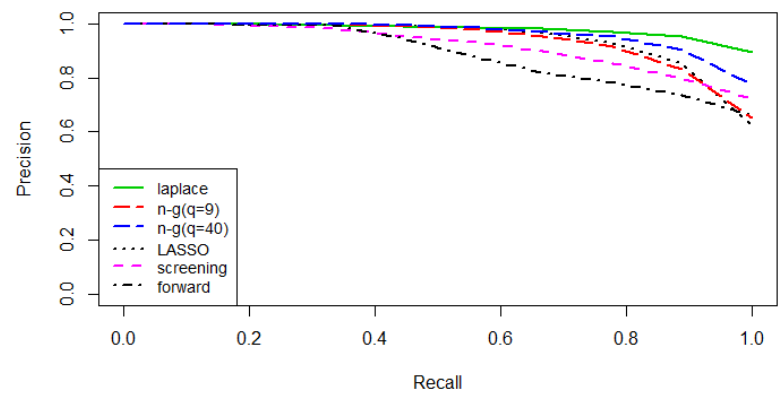

(a) PRC curve, $\rho=0.8$

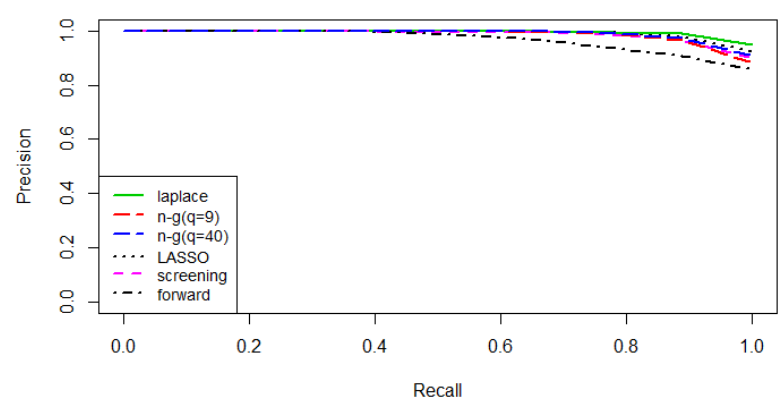

(c) PRC curve, $\rho=0.5$

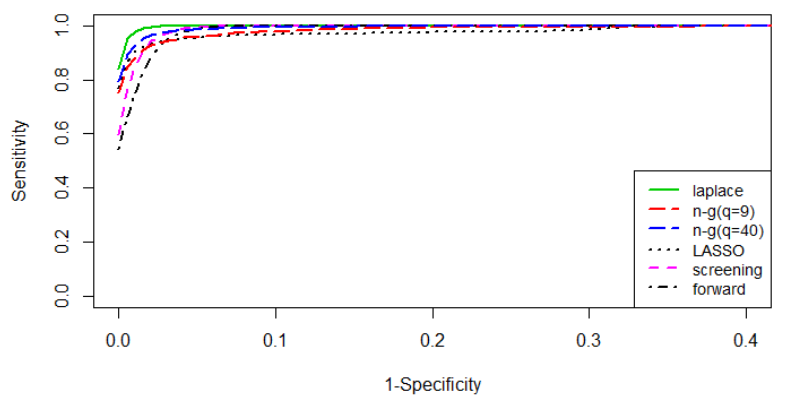

(b) ROC curve, $\rho=0.8$

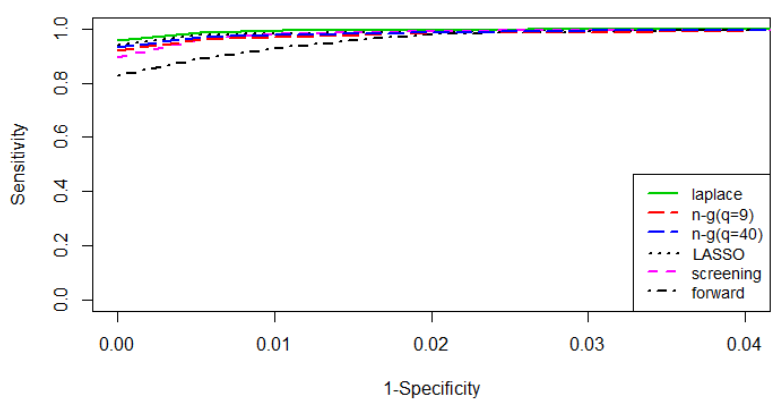

(d) ROC curve, $\rho=0.5$

Figure 6. Mean PRC and ROC curve from prospectively generating 250 datasets for $n=200$, $p=200, \rho=0.8,0.5$ with block signal.

covariance matrix by $\Sigma_{p \times p}(\rho)$. We generate $\boldsymbol{x}_{i} \mid y=1 \sim N\left(\boldsymbol{\mu}_{\mathbf{1}}, \Sigma_{p \times p}(\rho)\right)$, and $\boldsymbol{x}_{i} \mid y=0 \sim$ $N\left(\boldsymbol{\mu}_{\mathbf{0}}, \Sigma_{p \times p}(\rho)\right)$, with $\mu_{0}=\mathbf{0}_{p \times 1}$. We use an equal proportion of $y=0$ and $y=1$, with $\boldsymbol{y}=\left(\mathbf{1}_{n / 2}, \mathbf{0}_{n / 2}\right)$. The true $\beta_{j}, j=2, \ldots, p+1$ are given by $\Sigma^{-1}\left(\boldsymbol{\mu}_{\mathbf{1}}-\boldsymbol{\mu}_{\mathbf{0}}\right)$.

In order to generate sparse coefficients, we randomly choose three non-zero elements in the mean vector $\boldsymbol{\mu}_{\mathbf{1}}$. Due to the $\mathrm{AR}(1)$ correlation structure, $\Sigma^{-1}$ is a banded matrix, having non-zero elements on the diagonal and one step off of the diagonal. When multiplied by the mean vector, this results in a coefficient vector with 9 important vari- 


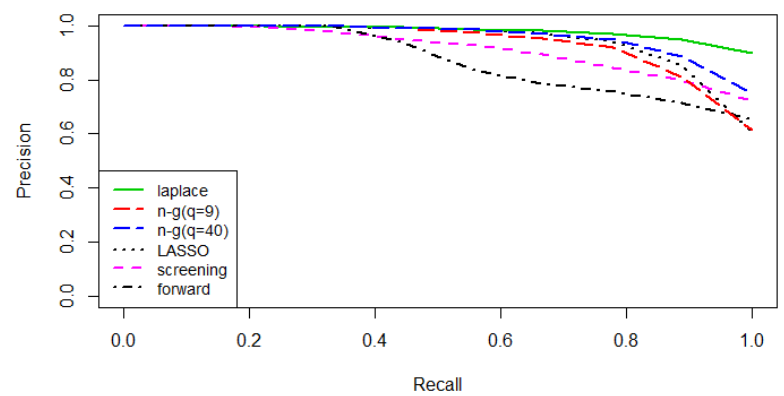

(a) PRC curve, $\rho=0.8, p=500$

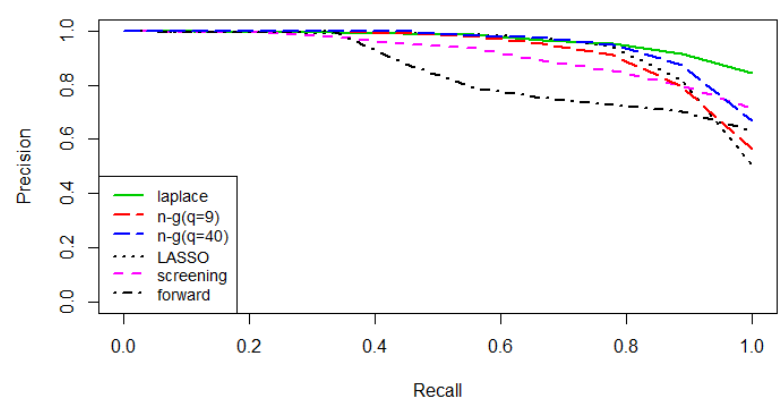

(c) PRC curve, $\rho=0.8, p=1000$

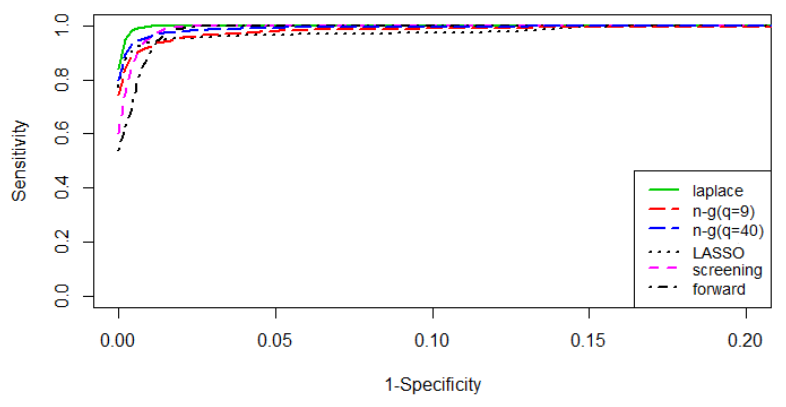

(b) ROC curve, $\rho=0.8, p=500$

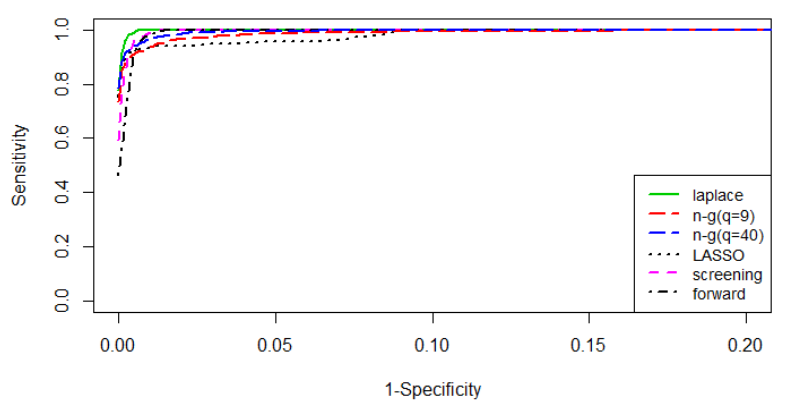

(d) ROC curve, $\rho=0.8, p=1000$

Figure 7. Mean PRC and ROC curve from prospectively generating 250 datasets for $n=200$, $p \in\{500,1000\}, \rho=0.8$ with block signal.

ables. With $\rho=0.5$, we use $\boldsymbol{\mu}_{\mathbf{1}}=\left(0,0,1, \mathbf{0}_{20}^{\prime}, 1, \mathbf{0}_{20}^{\prime}, 1, \mathbf{0}_{p-45}^{\prime}\right)$, which results in $\boldsymbol{\beta}=$ $\left(0,-1.33,2.73,-1.33, \mathbf{0}_{18},-1.33,2.73,-1.33, \mathbf{0}_{18},-1.33,2.73,-1.33, \mathbf{0}_{p-46}\right)$. For $\rho=0.8$, we use $\boldsymbol{\mu}_{\mathbf{1}}=\left(0,0,0.6, \mathbf{0}_{20}^{\prime}, 0.6, \mathbf{0}_{20}^{\prime}, 0.6, \mathbf{0}_{p-45}^{\prime}\right)$, which results in $\boldsymbol{\beta}=\left(0,-0.67,1.67,-0.67, \mathbf{0}_{18}\right.$, $\left.-0.67,1.67,-0.67, \mathbf{0}_{18},-0.67,1.67,-0.67, \mathbf{0}_{p-46}\right)$.

When data are generated retrospectively, the coefficients are created in blocks automatically according to the mean vector. It can be observed that although the important variables are formed in blocks, the induced adjacent variables have opposite signs. Therefore, the effect 
of adjacent variables may cancel each other out. We would expect similar selection result as in prospective data generation with spread signal.

Figure 8 plots the ROC and PRC curves for $p=200$. The upper two plots are for high correlation $(\rho=0.8)$ and lower two are for low correlation $(\rho=0.5)$. We observe that the Normal-Gamma prior tuned using $q=9$ performs best. The benefit of the joint credible region approach increases substantially for $\rho=0.8$. When the dimension of $p$ is increased to $p \in\{500,1000\}$, we see consistent results (Figure 9).

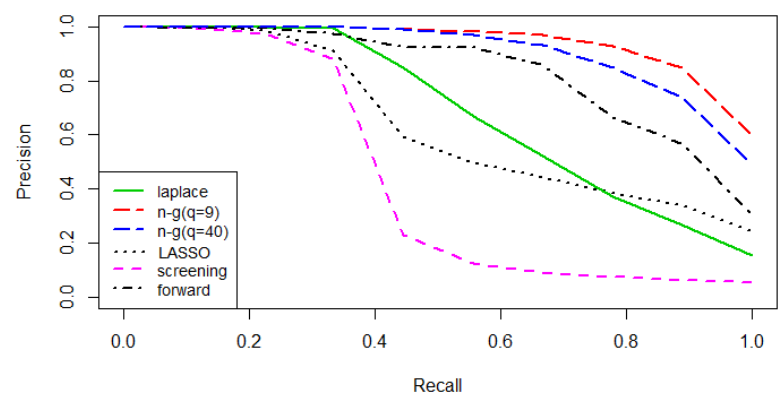

(a) PRC curve, $\rho=0.8$

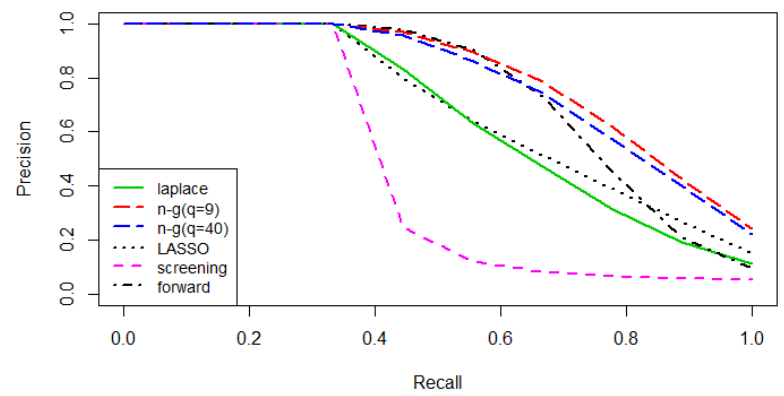

(c) PRC curve, $\rho=0.5$

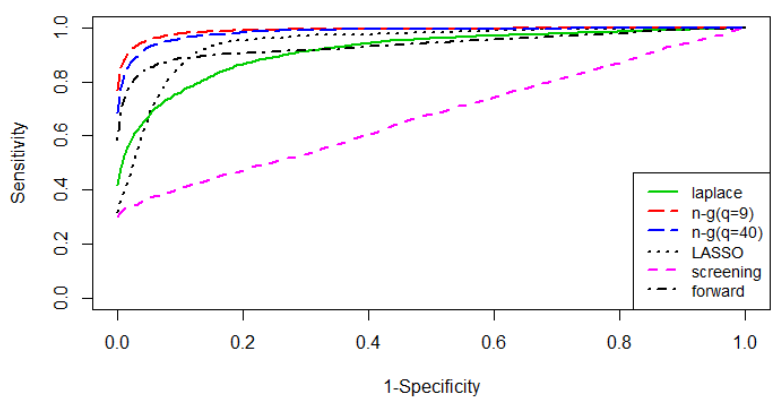

(b) ROC curve, $\rho=0.8$

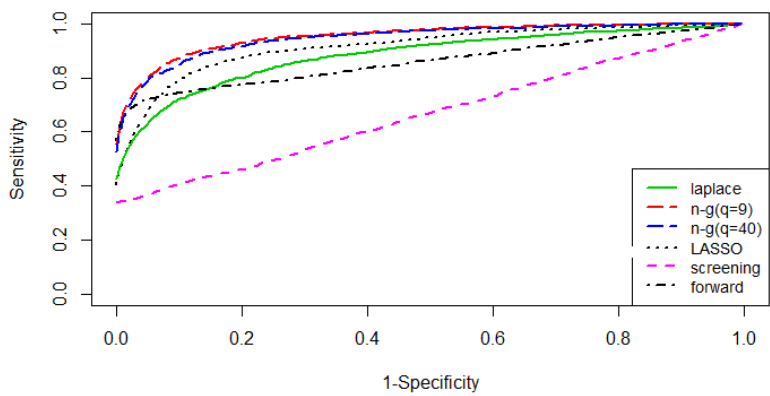

(d) ROC curve, $\rho=0.5$

Figure 8. Mean PRC and ROC curve from retrospectively generating 250 datasets for $n=$ $200, p=200, \rho=0.8,0.5$. 


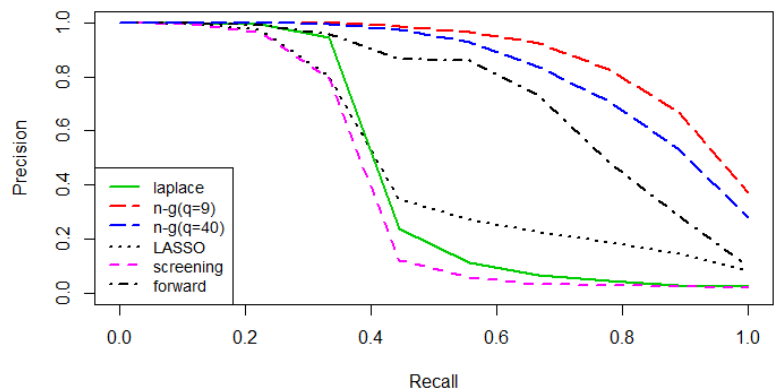

(a) PRC curve, $\rho=0.8, \mathrm{p}=500$

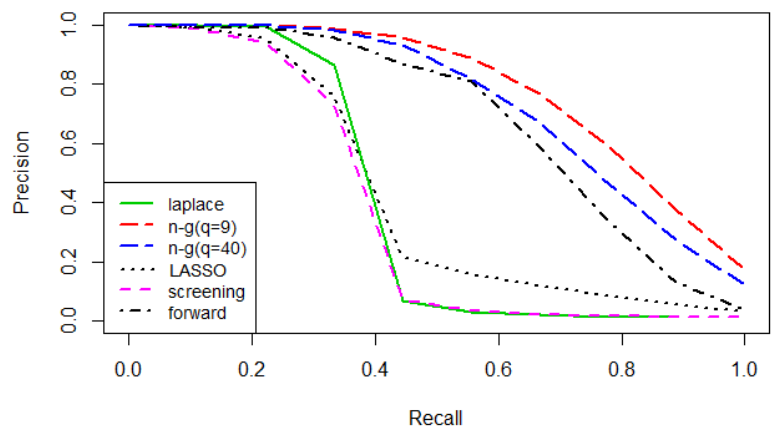

(c) PRC curve, $\rho=0.8, \mathrm{p}=1000$

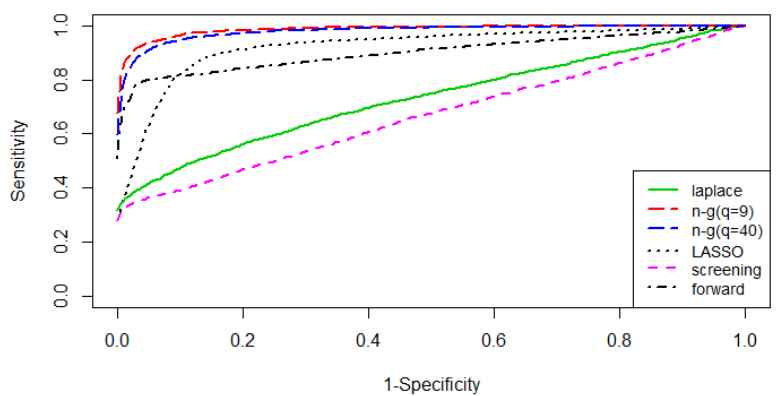

(b) ROC curve, $\rho=0.8, \mathrm{p}=500$

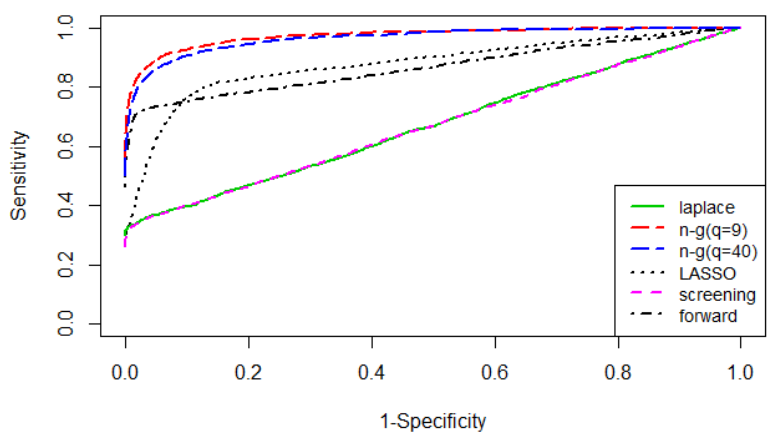

(d) ROC curve, $\rho=0.8, \mathrm{p}=1000$

Figure 9. Mean PRC and ROC curve from retrospectively generating 250 datasets for $n=$ $200, p \in\{500,1000\}, \rho=0.8$.

\subsection{Further Discussion of Adaptive Shrinkage}

From the simulation study, we observe that the adaptive shrinkage within the model is determined by both the data and the choice of prior. For the same degree of sparseness, the shrinkage can differ according to the data structure. In this section, we discuss the effect that the data structure and the prior choice have on the identification of important variables.

With the same number of important variables, we investigate two data structures. The 
first is "spread," where there is low to no correlation between the important predictors; the second is "blocking," where the important predictors are highly correlated. A tight prior, developed by tuning a Normal-Gamma distribution, tries to select a single variable from among several important variables that are highly correlated with each other (in a block). This will cause an error, as only one predictor will be selected. By contrast, if there is low correlation among the important variables, a tight prior would be able to make reasonable inference. On the other hand, since the Laplace prior is fairly smooth, it can be expected to work well when the important predictors are in blocks.

These effects can also be observed from the posterior distributions in Figure 10. This figure shows the posterior densities of two highly correlated predictors, $\beta_{1}$ and $\beta_{2}$, using both the Normal-Gamma and Laplace prior with $n=200, p=200$ under different data structures. The Normal-Gamma prior has $q=9$. Figure $10(\mathrm{a})$ and $10(\mathrm{~b})$ shows the spread case, where $\beta_{1}$ is important and $\beta_{2}$ is noise. From the figure, we observe that the Normal-Gamma prior gives support for $\beta_{1}$ being significantly different from 0 , while $\beta_{2}$ has been substantially shrunk toward 0. With the Laplace prior, since it is more smooth, the posterior modes for both $\beta_{1}$ and $\beta_{2}$ are shrunk towards zero. Figure 10(c) and 10(d) show the situation where the important variables are blocked together and both $\beta_{1}$ and $\beta_{2}$ are important. The NormalGamma prior selects only one of the important predictors, $\beta_{2}$; the posterior mode of $\beta_{1}$ has a peak around zero. However, the Laplace prior gives more even shrinkage to both of $\beta_{1}$ and $\beta_{2}$. In this case, the Laplace prior is preferred.

A tight prior implemented using a tuned Normal-Gamma prior is preferred if the important variables are likely to be spread out; otherwise, the Laplace prior is a better alternative. 


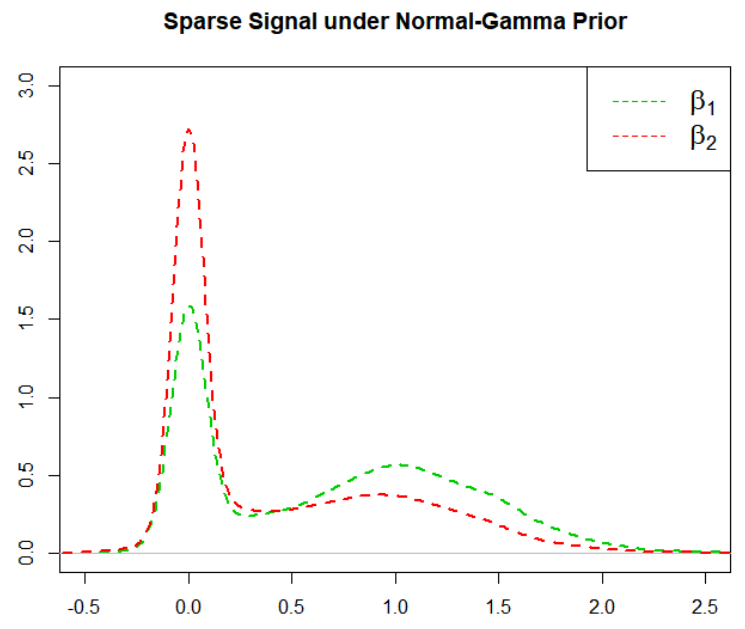

(a)

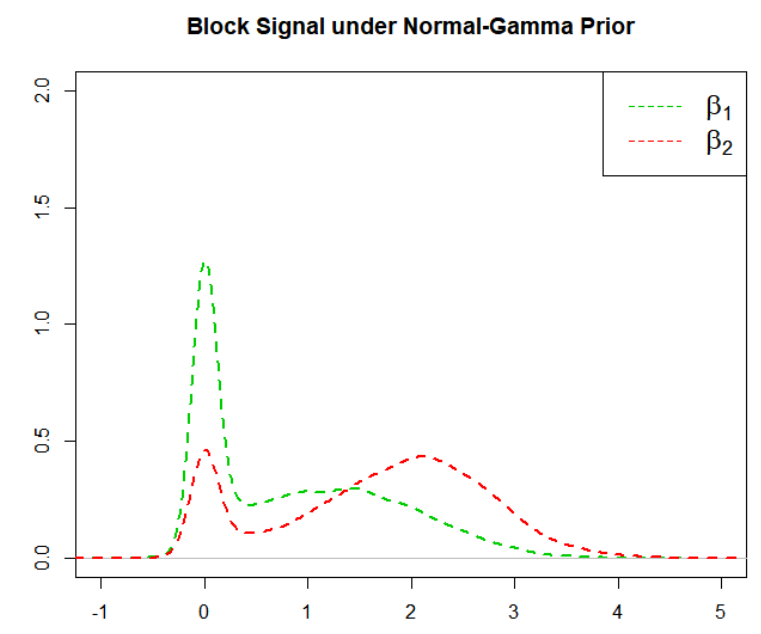

(c)

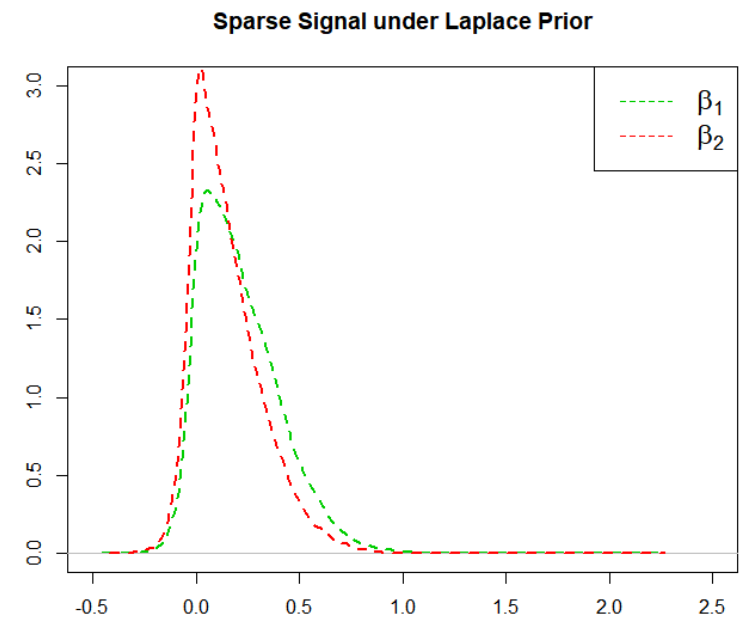

(b)

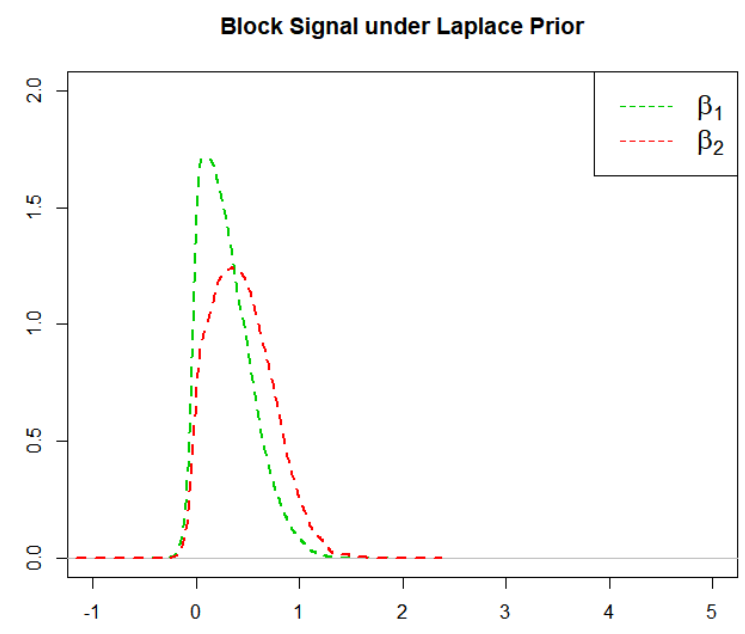

(d)

Figure 10. Effects of prior, sparse/block signal on posterior distributions.

\subsection{Model Selection via AIC/BIC}

In the previous simulation studies, we considered the entire solution path for each method. However, in practice, we want to use the variable selection method to choose a reduced set 
of variables. For each method, we considered AIC and BIC as criteria for selecting a reduced set of variables and ran an additional set of simulations. We consider both $q=9$ and $q=3$ important predictors. The results for $p \in\{200,1000\}$ and $\rho=0.8$, based on 250 datasets, are shown in Tables 2-5, which report the coverage proportion (COV; the proportion of times that the selected model covers the true model), average number of important predictors included (IP), and average number of unimportant predictors included (UP). Note that as the size of the model increases, there would be an increase in both IP and UP, since model size is the sum of the two. Hence there is a tradeoff, so a desirable method would have a large IP, but small UP. In each column of the tables, we highlight the largest values for COV and IP, and the smallest values for UP.

From Tables 2-5, we can see that for either $q=9$ or $q=3$, AIC includes more predictors in the subset than BIC. With $p=200$, the results show that with spread signals, in most cases, the joint credible region with a tight prior has better selection properties among all the other methods as measured by smaller number of uninformative predictors, larger coverage proportion, and average number of important predictors. Screening performs the worst, with more uninformative predictors and fewer important predictors. With block signals, as expected, the Laplace prior displays best selection properties, while forward selection does worse, with low coverage proportion and average important predictors.

When we increase the dimension to $p=1000$, the selection results are consistent with the $p=200$ case for both the sparse and block case. Overall, the selection results are consistent with the variable selection results.

These conclusions are based on what we know as "truth": the data structure. However, in reality, we do not know which variables are important, how they are distributed in the 
Table 2. BIC- and AIC-based selection performance with sparse signal for $n=200, \mathrm{p}$ $\in\{200,1000\}$ based on 250 datasets. The entries in the table are coverage proportion (COV), average number of important predictors out of the 9 included (IP), and average number of unimportant predictors (UP) out of $p-9$.

\begin{tabular}{|c|c|c|c|c|c|c|c|c|c|c|c|c|}
\hline & \multicolumn{6}{|c|}{$\mathrm{BIC}$} & \multicolumn{6}{|c|}{$\mathrm{AIC}$} \\
\hline & \multicolumn{3}{|c|}{$\mathrm{p}=200$} & \multicolumn{3}{|c|}{$\mathrm{p}=1000$} & \multicolumn{3}{|c|}{$\mathrm{p}=200$} & \multicolumn{3}{|c|}{$\mathrm{p}=1000$} \\
\hline & $\mathrm{COV}$ & IP & UP & $\mathrm{COV}$ & IP & UP & $\mathrm{COV}$ & IP & UP & $\mathrm{COV}$ & IP & UP \\
\hline$n-g(q=9)$ & 49.2 & 8.22 & 1.96 & 39.2 & 7.97 & 7.03 & 70.8 & 8.64 & 6.1 & 44.0 & 8.18 & 8.38 \\
\hline$n-g(q=40)$ & 47.6 & 8.21 & 2.67 & 29.6 & 7.68 & 5.99 & 73.6 & 8.66 & 6.45 & 38.0 & 8.09 & 8.23 \\
\hline Laplace & 14.4 & 7.29 & 3.87 & 2.4 & 5.6 & 6.58 & 29.6 & 7.9 & 6.58 & 1.6 & 6.33 & 9.64 \\
\hline Tuned n-g & 53.6 & 8.28 & 3.15 & 31.6 & 7.82 & 5.8 & 74.0 & 8.66 & 6.44 & 44.0 & 8.19 & 8.45 \\
\hline Forward Selection & 29.6 & 7.59 & 4.83 & 9.6 & 6.47 & 5.63 & 34.8 & 7.74 & 5.79 & 14.8 & 6.67 & 7.15 \\
\hline Screening & 2.0 & 4.8 & 7.48 & 0.0 & 4.39 & 6.23 & 4.0 & 6.7 & 17.3 & 0.0 & 5.13 & 10.33 \\
\hline LASSO & 29.6 & 7.59 & 5.36 & 30.8 & 7.6 & 8.38 & 46.8 & 8.19 & 7.58 & 31.2 & 7.82 & 9.46 \\
\hline
\end{tabular}

data set, or the correlation between the important variables. Consequently, we suggest a grid tuning process for $q$. We may pick a few values of $q$, then compute the AIC/BIC along the solution path for each value of $q$. Then we choose the solution that gives the minimum AIC/BIC, i.e. we tune on a 2-d grid. In Tables 2-5, the "Tuned n-g" row gives the selection results with the tuned $q$ for each data set based on $q \in\{9,40,140\}$. It shows that, with the tuned $q$, although it may not be better than using the "truth", in most cases, it still outperforms the other Non-Bayesian methods, and performs reasonably well relative to using the known $q$. 
Table 3. BIC- and AIC-based selection performance with sparse signal for $n=200, \mathrm{p}$ $\in\{200,1000\}$ based on 250 datasets. The entries in the table denote coverage proportion (COV), average number of important predictors out of the 3 included (IP), and average number of unimportant predictors (UP) out of $p-9$.

\begin{tabular}{|c|c|c|c|c|c|c|c|c|c|c|c|c|}
\hline & \multicolumn{6}{|c|}{$\mathrm{BIC}$} & \multicolumn{6}{|c|}{$\mathrm{AIC}$} \\
\hline & \multicolumn{3}{|c|}{$\mathrm{p}=200$} & \multicolumn{3}{|c|}{$\mathrm{p}=1000$} & \multicolumn{3}{|c|}{$\mathrm{p}=200$} & \multicolumn{3}{|c|}{$\mathrm{p}=1000$} \\
\hline & $\mathrm{COV}$ & IP & UP & $\mathrm{COV}$ & IP & $\mathrm{UP}$ & $\mathrm{COV}$ & IP & UP & $\mathrm{COV}$ & IP & UP \\
\hline$n-g(q=3)$ & 99.6 & 2.99 & 2.67 & 99.2 & 2.99 & 4.6 & 100.0 & 3 & 9.06 & 100.0 & 3 & 5.72 \\
\hline$n-g(q=40)$ & 97.6 & 2.97 & 1.73 & 98.8 & 2.99 & 3.88 & 100.0 & 3 & 8.16 & 100.0 & 3 & 5.54 \\
\hline Laplace & 94.8 & 2.9 & 0.37 & 95.2 & 2.95 & 1.15 & 99.2 & 2.99 & 5.01 & 99.6 & 2.99 & 1.91 \\
\hline Tuned n-g & 99.2 & 2.99 & 2.45 & 99.2 & 2.99 & 4.66 & 100.0 & 3 & 5.46 & 100.0 & 3 & 5.75 \\
\hline Forward Selection & 96.0 & 2.96 & 6.94 & 93.6 & 2.93 & 5.79 & 100.0 & 2.96 & 10.35 & 96.0 & 2.96 & 8.53 \\
\hline Screening & 86.8 & 2.86 & 3.33 & 72.0 & 2.69 & 2.47 & 97.2 & 2.97 & 4.63 & 99.6 & 2.99 & 5.58 \\
\hline LASSO & 96.8 & 2.97 & 1.13 & 98.8 & 2.98 & 3.12 & 100.0 & 3 & 9.65 & 100.0 & 3 & 13.21 \\
\hline
\end{tabular}


Table 4. BIC- and AIC-based selection performance with block signal for $n=200, \mathrm{p} \in$ $\{200,1000\}$ based on 250 datasets. The entries in the table are coverage proportion (COV), average number of important predictors out of the 9 included (IP), and average number of unimportant predictors (UP) out of $p-9$.

\begin{tabular}{|c|c|c|c|c|c|c|c|c|c|c|c|c|}
\hline & \multicolumn{6}{|c|}{$\mathrm{BIC}$} & \multicolumn{6}{|c|}{$\mathrm{AIC}$} \\
\hline & \multicolumn{3}{|c|}{$p=200$} & \multicolumn{3}{|c|}{$\mathrm{p}=1000$} & \multicolumn{3}{|c|}{$\mathrm{p}=200$} & \multicolumn{3}{|c|}{$\mathrm{p}=1000$} \\
\hline & $\mathrm{COV}$ & IP & UP & $\mathrm{COV}$ & IP & UP & $\mathrm{COV}$ & IP & UP & $\mathrm{COV}$ & IP & UP \\
\hline$n-g(q=9)$ & 38.4 & 7.8 & 4.14 & 25.2 & 7.48 & 2.57 & 56.0 & 8.41 & 6.38 & 36.4 & 8.1 & 4.02 \\
\hline $\mathrm{n}-\mathrm{g}(\mathrm{q}=40)$ & 42.0 & 7.84 & 4.09 & 32.4 & 7.54 & 1.9 & 57.6 & 8.44 & 6.27 & 52.8 & 8.4 & 3.64 \\
\hline Laplace & 46.0 & 7.7 & 1.78 & 29.6 & 7.6 & 0.69 & 88.0 & 8.86 & 5.61 & 72.8 & 8.64 & 1.95 \\
\hline Tuned n-g & 40.8 & 7.84 & 4.06 & 29.2 & 7.59 & 2.69 & 55.2 & 8.4 & 6.1 & 42.0 & 8.2 & 3.98 \\
\hline Forward Selection & 17.6 & 6.59 & 2.84 & 7.6 & 5.58 & 2.57 & 61.6 & 8.24 & 6.01 & 24.0 & 7.39 & 3.48 \\
\hline Screening & 42.4 & 8 & 3.25 & 19.6 & 7.43 & 1.62 & 77.2 & 8.74 & 5.4 & 54.0 & 8.3 & 2.87 \\
\hline LASSO & 44.8 & 7.95 & 4.44 & 23.2 & 7.49 & 2.07 & 60.0 & 8.51 & 6.92 & 40.0 & 8.2 & 4.08 \\
\hline
\end{tabular}


Table 5. BIC- and AIC-based selection performance with block signal for $n=200, \mathrm{p} \in$ $\{200,1000\}$ based on 250 datasets. The entries in the table are coverage proportion (COV), average number of important predictors out of the 3 included (IP), and average number of unimportant predictors (UP) out of $p-9$.

\begin{tabular}{|c|c|c|c|c|c|c|c|c|c|c|c|c|}
\hline & \multicolumn{6}{|c|}{$\mathrm{BIC}$} & \multicolumn{6}{|c|}{$\mathrm{AIC}$} \\
\hline & \multicolumn{3}{|c|}{$\mathrm{p}=200$} & \multicolumn{3}{|c|}{$\mathrm{p}=1000$} & \multicolumn{3}{|c|}{$\mathrm{p}=200$} & \multicolumn{3}{|c|}{$\mathrm{p}=1000$} \\
\hline & $\mathrm{COV}$ & IP & UP & $\mathrm{COV}$ & IP & UP & $\mathrm{COV}$ & IP & UP & $\mathrm{COV}$ & IP & UP \\
\hline$n-g(q=3)$ & 79.6 & 2.79 & 1.61 & 88.4 & 2.88 & 4.19 & 92.4 & 2.94 & 2.74 & 93.6 & 2.94 & 5.76 \\
\hline $\mathrm{n}-\mathrm{g}(\mathrm{q}=40)$ & 84.8 & 2.85 & 1.34 & 93.6 & 2.94 & 3.86 & 96.8 & 2.97 & 2.63 & 97.6 & 2.98 & 5.6 \\
\hline Laplace & 84.0 & 2.84 & 0.36 & 88.8 & 2.88 & 0.34 & 99.2 & 2.99 & 1.77 & 99.2 & 2.99 & 4.15 \\
\hline Tuned n-g & 80.4 & 2.80 & 1.68 & 90.4 & 2.90 & 4.14 & 93.2 & 2.93 & 2.81 & 94.0 & 2.94 & 5.82 \\
\hline Forward Selection & 74.8 & 2.73 & 2.67 & 60.8 & 2.57 & 4.43 & 77.2 & 2.76 & 3.17 & 76.0 & 2.74 & 4.71 \\
\hline Screening & 86.0 & 2.86 & 0.38 & 81.6 & 2.82 & 0.32 & 98.0 & 2.98 & 0.76 & 97.6 & 2.98 & 1.84 \\
\hline LASSO & 92.4 & 2.92 & 1.15 & 98.0 & 2.98 & 3.84 & 98.8 & 2.98 & 2.7 & 98.8 & 2.98 & 5.7 \\
\hline
\end{tabular}




\section{Discussion and Conclusions}

Overall, variable selection using the joint credible region approach results in a better performance than other methods, as measured by ROC and PRC curves, for both low and high dimensional cases. The improvement of the joint credible region method over existing methods increases significantly with higher correlation. Specifically, in the situation when data are generated prospectively with a spread signal, we would expect a joint credible region approach with a tight prior to work well, followed by forward selection. The poor performance of screening is due to the low marginal correlation. When the signals are in blocks, the selection results reverse. In this case, we would expect the smooth priors to perform best, while forward selection does poorly. Retrospectively generated data shows similar selection properties as the spread signal case; again, we expect the tight prior and forward selection do the best. This is because the opposite signs of the important variables enable cancellation of much of effect of the adjacent signals.

These results are sensitive to the choice of prior and hyper-parameters. The block signals are better detected using the Laplace prior, while the spread signals are better detected using

a tight prior. In reality, since we do not know which variables are important, we try both priors to see if they give similar results. If not, we rely on expert knowledge to make the selection. 


\section{References}

[1] H. Akaike. A new look at the statistical model identification. IEEE Transactions on Automatic Control, 19(6):716-723, 1974.

[2] Howard D. Bondell and Brian J. Reich. Consistent high-dimensional Bayesian variable selection via penalized credible regions. Journal of the American Statistical Association, 107(500):1610-1624, 2012.

[3] Emmanuel Candes and Terence Tao. The Dantzig selector: Statistical estimation when p is much larger than n. Annals of Statistics, 35(6):2313-2351, 2007.

[4] Petros Dellaportas, Jonathan J. Forster, and Ioannis Ntzoufras. On Bayesian model and variable selection using MCMC. Statistics and Computing, 12(1):27-36, 2002.

[5] B. Efron, T. Hastie, I. Johnstone, and R. Tibshirani. Least angle regression. Annals of Statistics, 32(2):407-499, 2004.

[6] Jianqing Fan and Runze Li. Variable selection via nonconcave penalized likelihood and its oracle properties. Journal of the American Statistical Association, 96(456):13481360, 2001.

[7] J Friedman, T Hastie, and R Tibshirani. Regularization paths for generalized linear models via coordinate descent. Journal of Statistical Software, 33(1):1-22, 2010.

[8] Lingrui Gan, Naveen Narisetty, and Feng Liang. Bayesian regularization for graphical models with unequal shrinkage. Journal of the American Statistical Association, 2018. To appear. 
[9] Edward I. George and Robert E. McCulloch. Variable selection via Gibbs sampling. Journal of the American Statistical Association, 88(423):881-889, 1993.

[10] Steven N Goodman. Toward evidence-based medical statistics. 2: The Bayes factor. Annals of Internal Medicine, 130(12):1005-1013, 1999.

[11] Jim E. Griffin and Philip J. Brown. Inference with normal-gamma prior distributions in regression problems. Bayesian Analysis, 5(1):171-188, 2010.

[12] Timothy E. Hanson, Adam J. Branscum, and Wesley O. Johnson. Informative $g$-priors for logistic regression. Bayesian Analysis, 9(3):597-612, 2014.

[13] Harold Jeffreys. An invariant form for the prior probability in estimation problems. Proceedings of the Royal Society of London, Series A, Mathematical and Physical Sciences, 186(1007):453-461, 1946.

[14] R. E Kass and A. E Raftery. Bayes factors. Journal of the American Statistical Association, 90(430):773-795, 1995.

[15] Trevor Park and George Casella. The Bayesian Lasso. Journal of the American Statistical Association, 103(482):681-686, 2008.

[16] Nicholas G. Polson, James G. Scott, and Jesse Windle. Bayesian inference for logistic models using Pólya-gamma latent variables. Journal of the American Statistical Association, 108(504):1339-1349, 2013.

[17] Veronika Rockova. Bayesian estimation of sparse signals with a continuous spike-andslab prior. Annals of Statistics, 46(1):401-437, 2018. 
[18] Veronika Rockova and Edward George. The spike-and-slab LASSO. Journal of the American Statistical Association, 113(521):431-444, 2018.

[19] Gideon Schwarz. Estimating the dimension of a model. Annals of Statistics, 6(2): 461-464, 1978 .

[20] R. Tibshirani. Regression shrinkage and selection via the LASSO. Journal of the Royal Statistical Society (Series B), 58:267-288, 1996.

[21] Ming Yuan and Yi Lin. Model selection and estimation in regression with grouped variables. Journal of the Royal Statistical Society (Series B), 68:49-67, 2006.

[22] Dietmar Zellner, Frieder Keller, and GÃijnter E. Zellner. Variable selection in logistic regression models. Communications in Statistics - Simulation and Computation, 33(3): 787-805, 2004.

[23] Hui Zou. The adaptive LASSO and its oracle properties. Journal of the American Statistical Association, 101(476):1418-1429, 2006.

[24] Hui Zou and Trevor Hastie. Regularization and variable selection via the elastic net. Journal of the Royal Statistical Society: Series B (Statistical Methodology), 67(2):301$320,2005$.

[25] Hui Zou and Runze Li. One-step sparse estimates in nonconcave penalized likelihood models. Annals of Statistics, 36(4):1509-1533, 2008. 
Block Signal under Laplace Prior

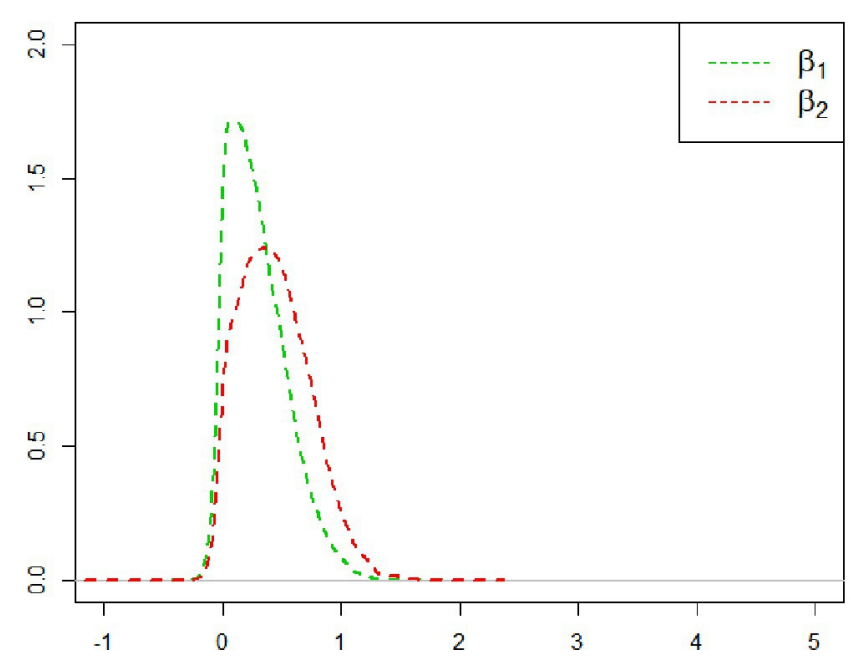

sam_11428_b_lp.eps

This article is protected by copyright. All rights reserved. 


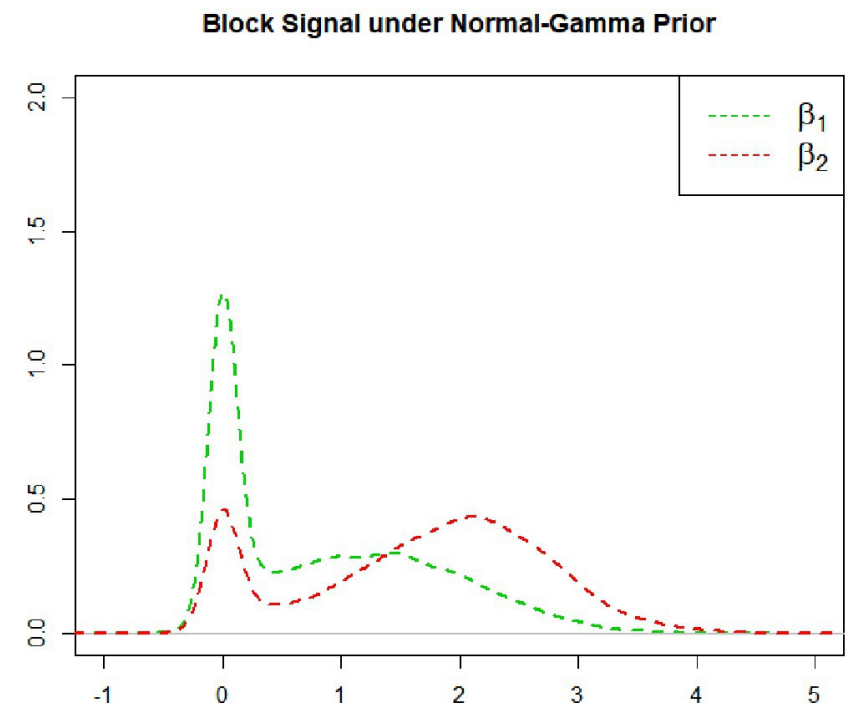

sam_11428_b_ng.eps

This article is protected by copyright. All rights reserved. 


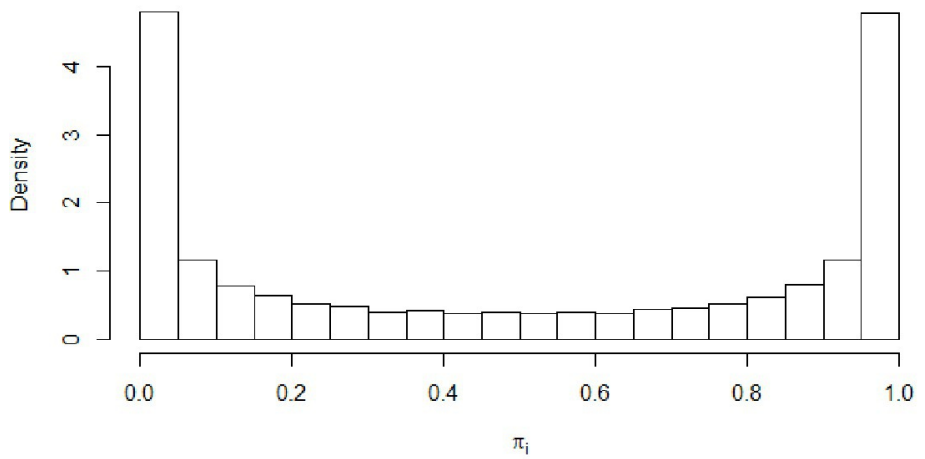

sam_11428_c1.eps

This article is protected by copyright. All rights reserved. 


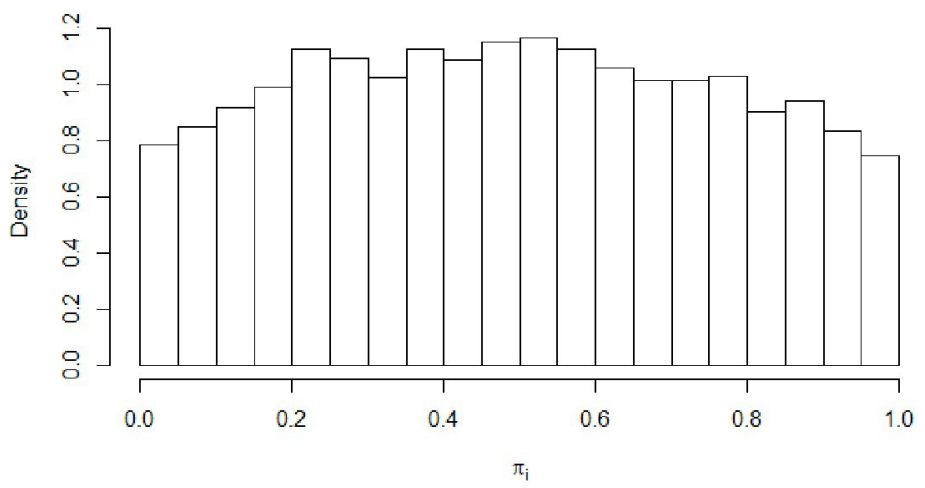

sam_11428_c2.eps

This article is protected by copyright. All rights reserved. 


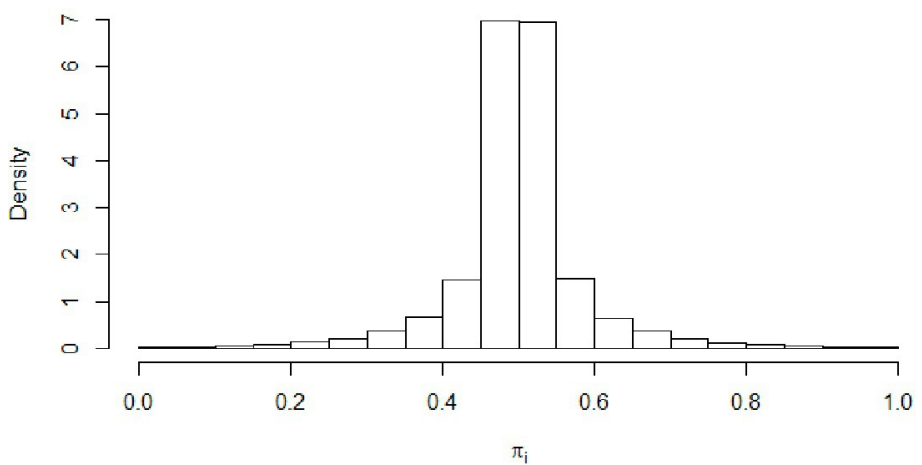

sam_11428_c3.eps

This article is protected by copyright. All rights reserved. 
Spread

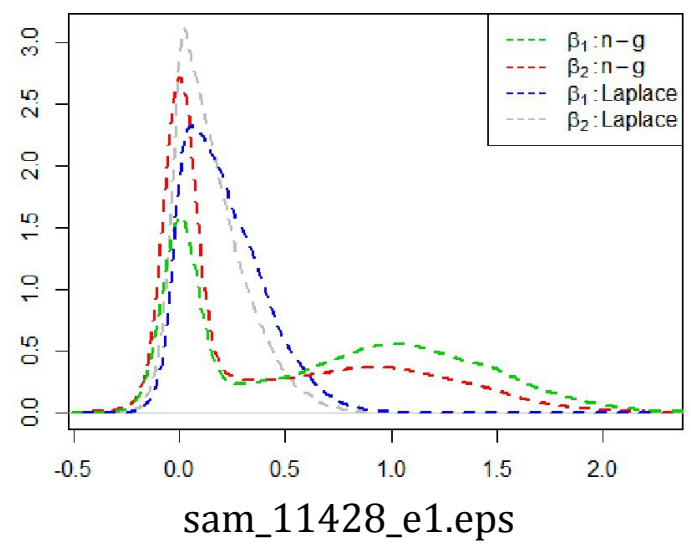

This article is protected by copyright. All rights reserved. 


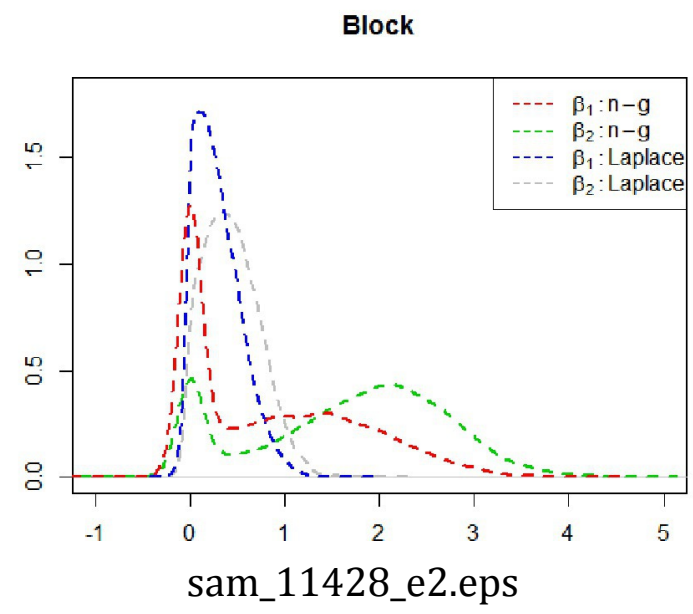

This article is protected by copyright. All rights reserved. 


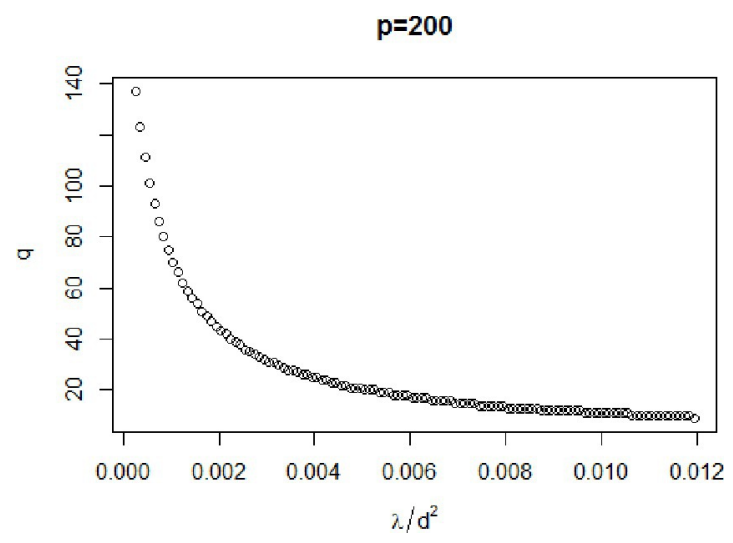

sam_11428_frac200.eps

This article is protected by copyright. All rights reserved. 


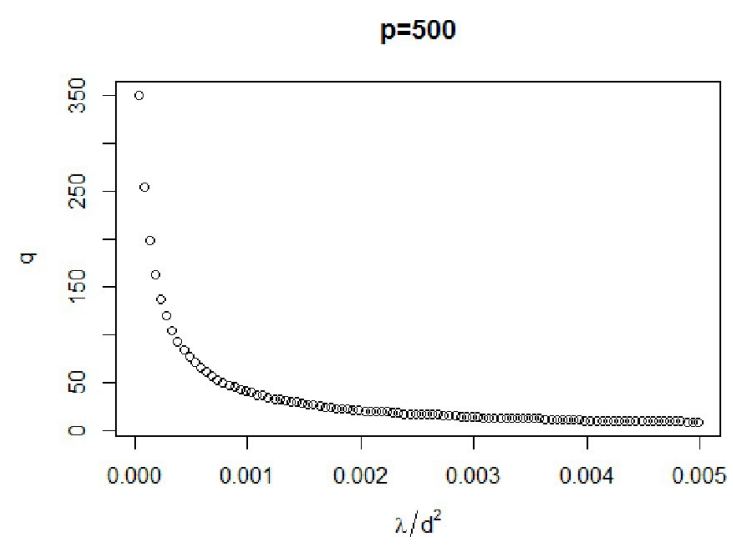

sam_11428_frac500.eps

This article is protected by copyright. All rights reserved. 


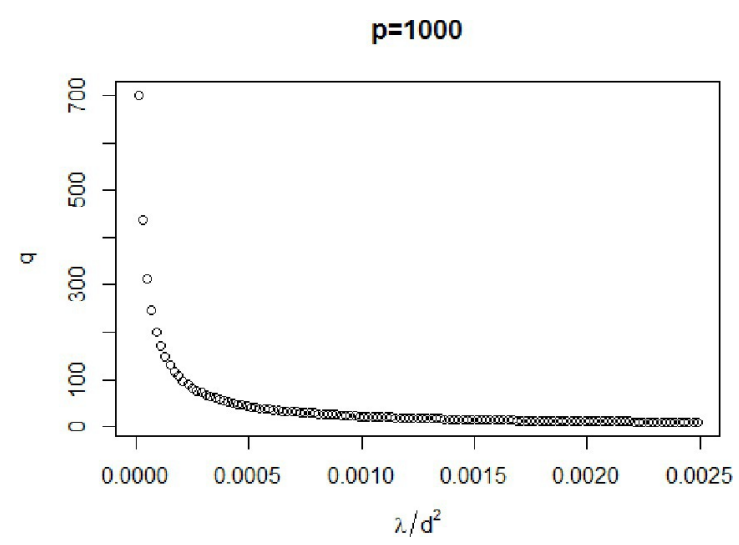

sam_11428_frac1000.eps

This article is protected by copyright. All rights reserved. 
$\operatorname{PRC}(200,200,0.8)$, a

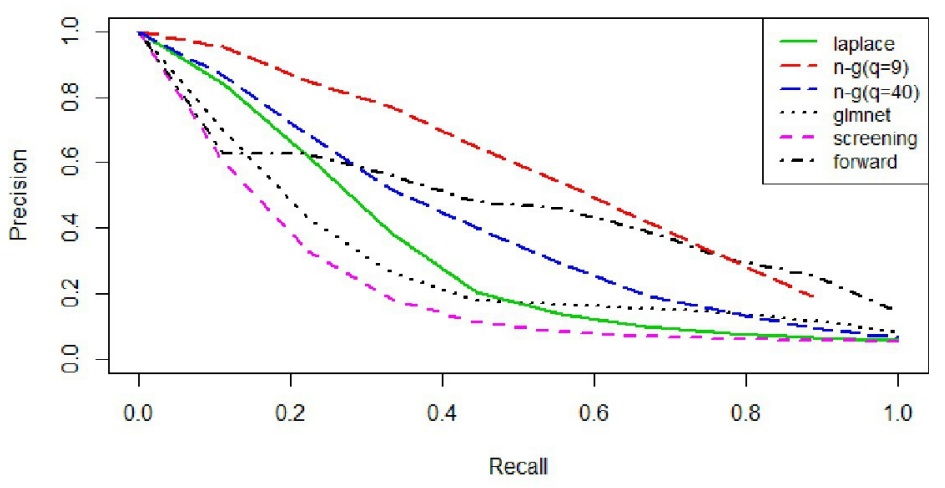

sam_11428_l1.eps

This article is protected by copyright. All rights reserved. 


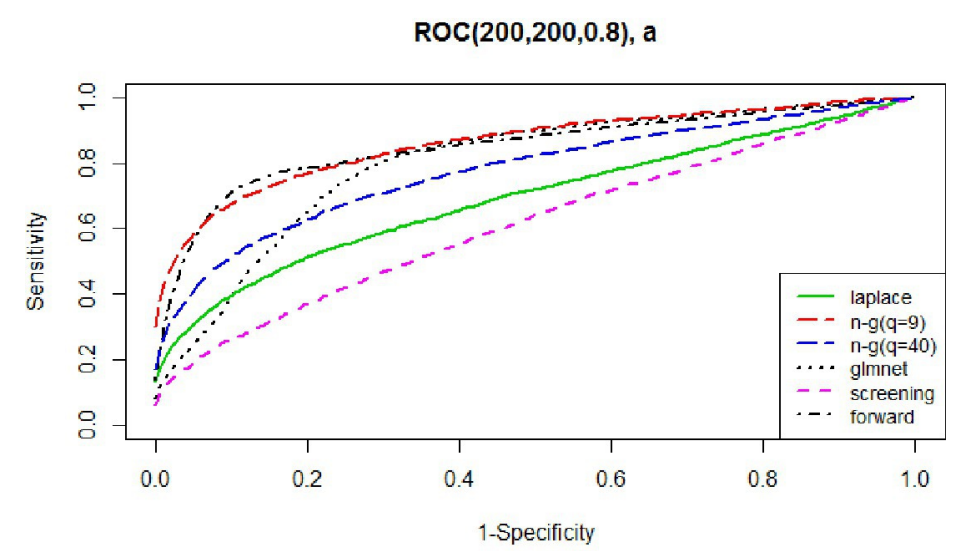

sam_11428_12.eps

This article is protected by copyright. All rights reserved. 


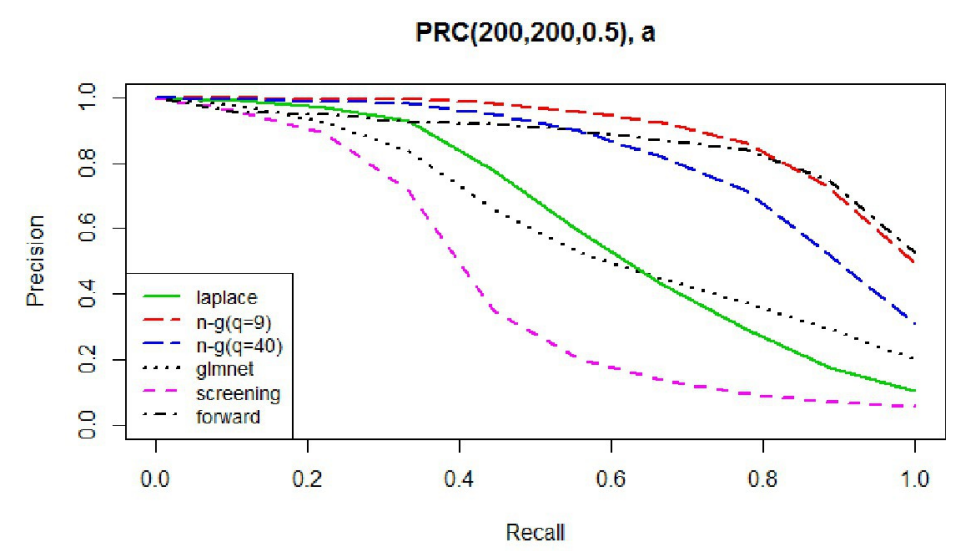

sam_11428_13.eps

This article is protected by copyright. All rights reserved. 


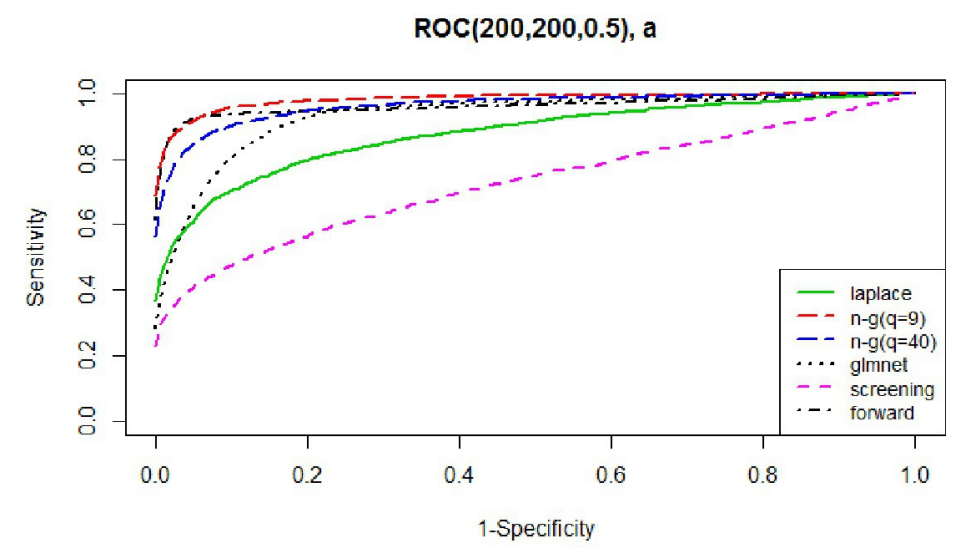

sam_11428_14.eps

This article is protected by copyright. All rights reserved. 
$\operatorname{PRC}(200,500,0.8)$, a

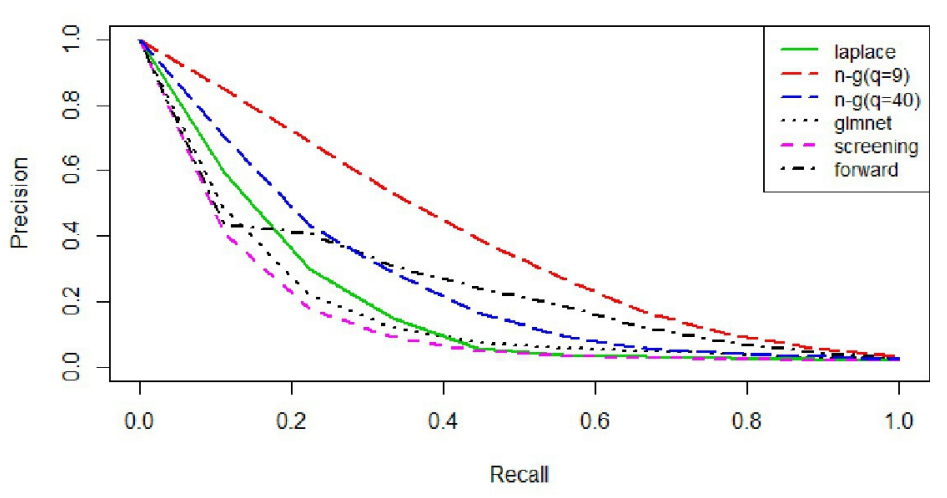

sam_11428_15.eps

This article is protected by copyright. All rights reserved. 


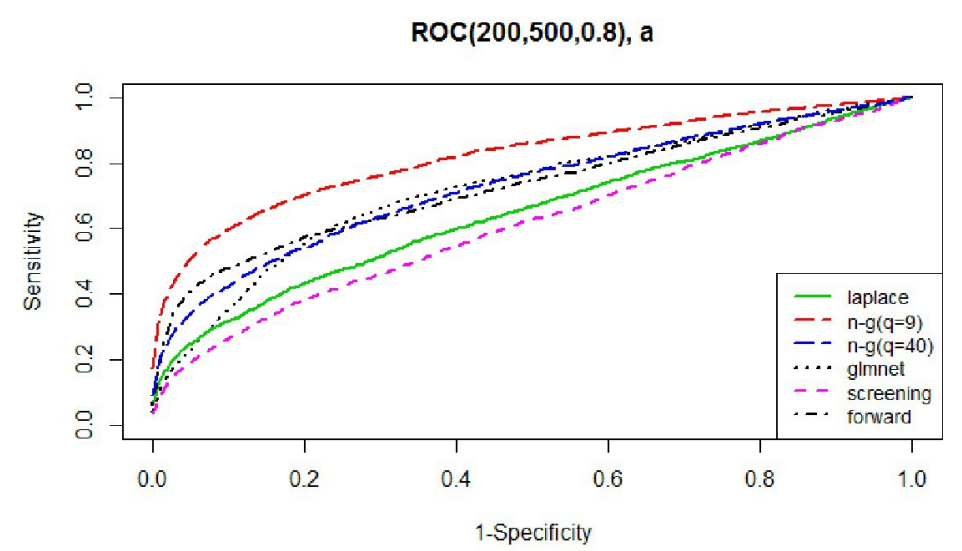

sam_11428_16.eps

This article is protected by copyright. All rights reserved. 


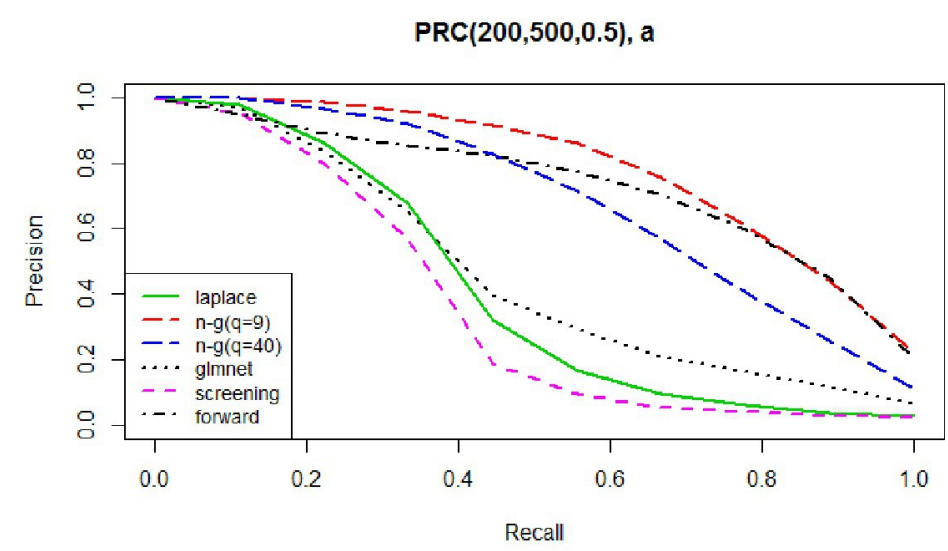

sam_11428_17.eps

This article is protected by copyright. All rights reserved. 


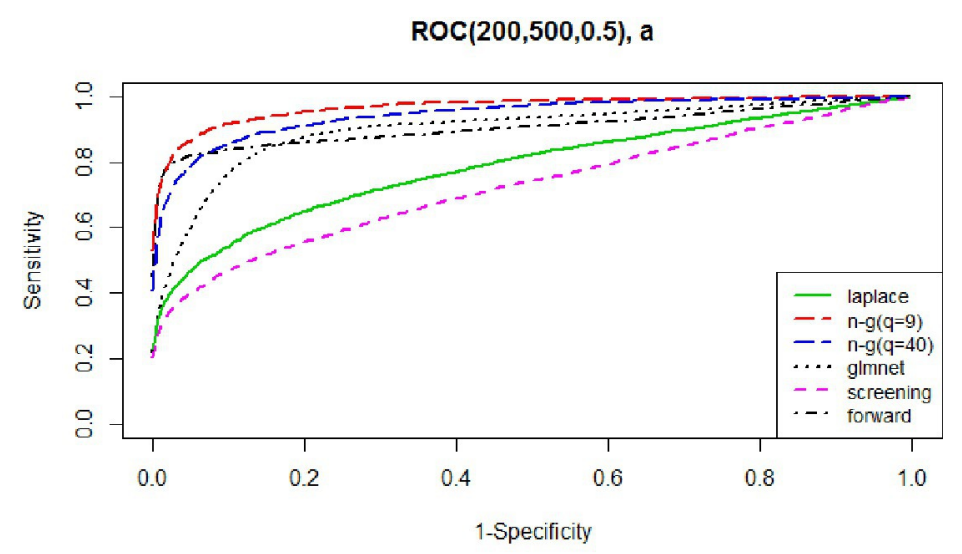

sam_11428_18.eps

This article is protected by copyright. All rights reserved. 


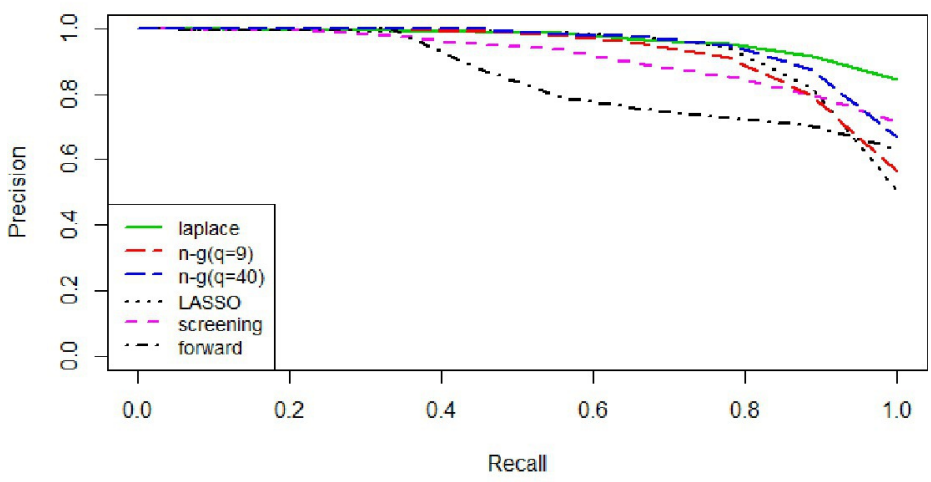

sam_11428_m1.eps

This article is protected by copyright. All rights reserved. 


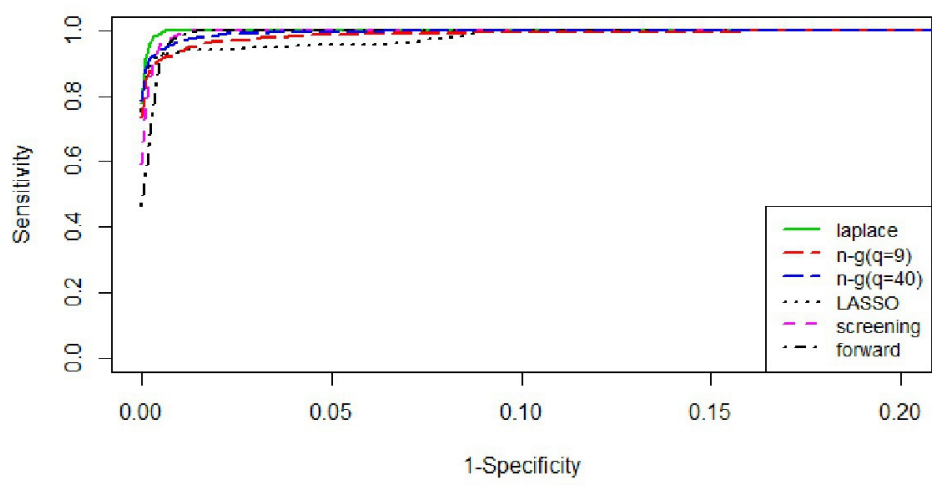

sam_11428_m2.eps

This article is protected by copyright. All rights reserved. 


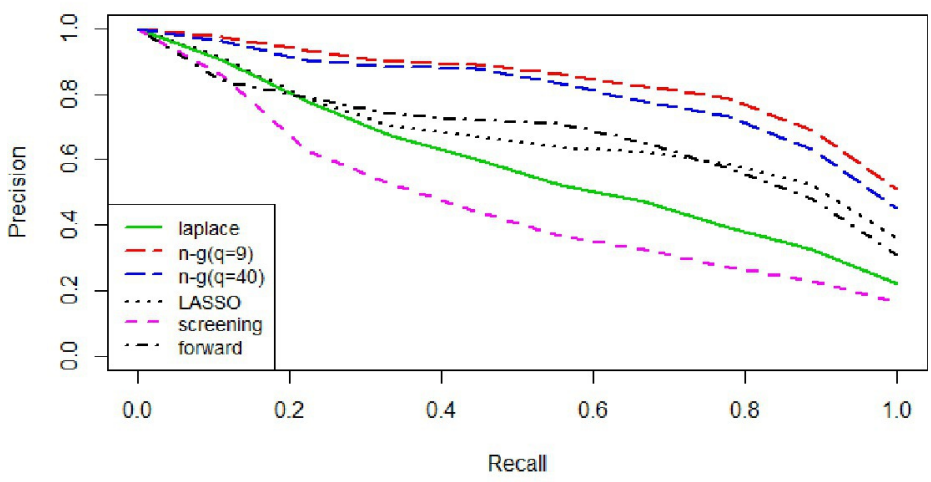

sam_11428_m5.eps

This article is protected by copyright. All rights reserved. 


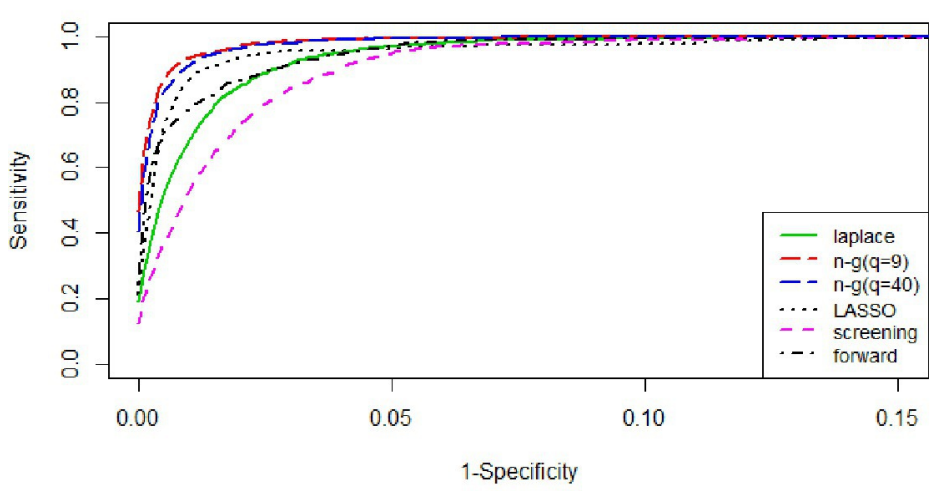

sam_11428_m6.eps

This article is protected by copyright. All rights reserved. 


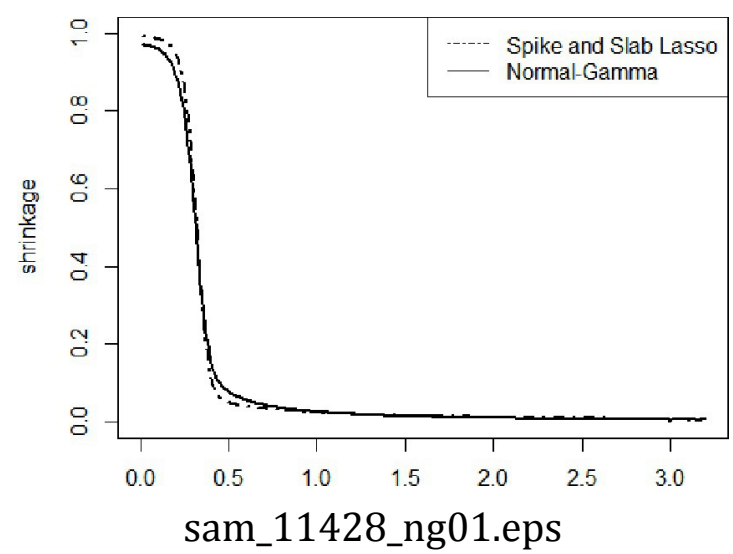

This article is protected by copyright. All rights reserved. 


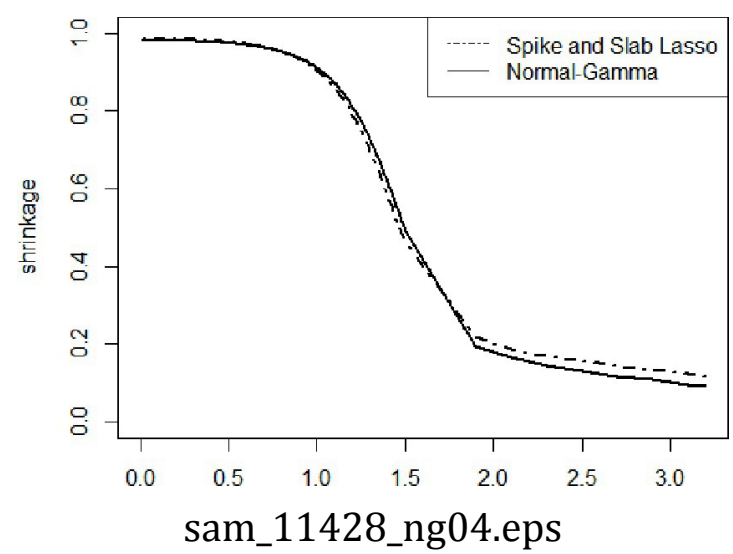

This article is protected by copyright. All rights reserved. 


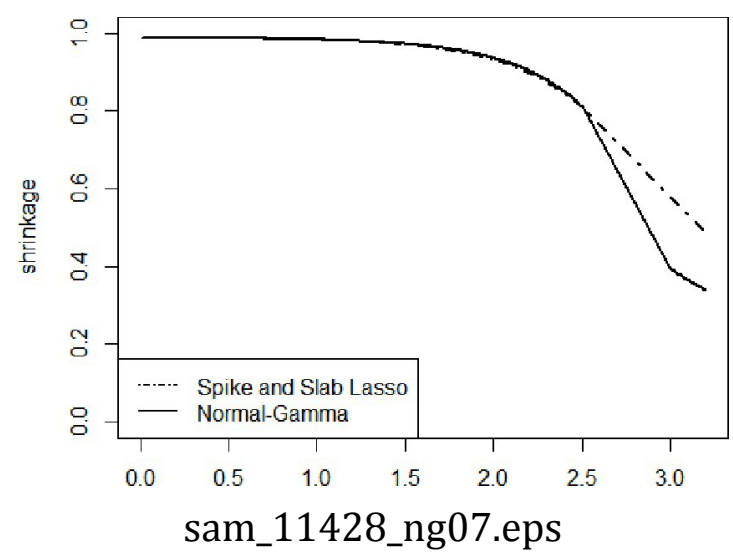

This article is protected by copyright. All rights reserved. 


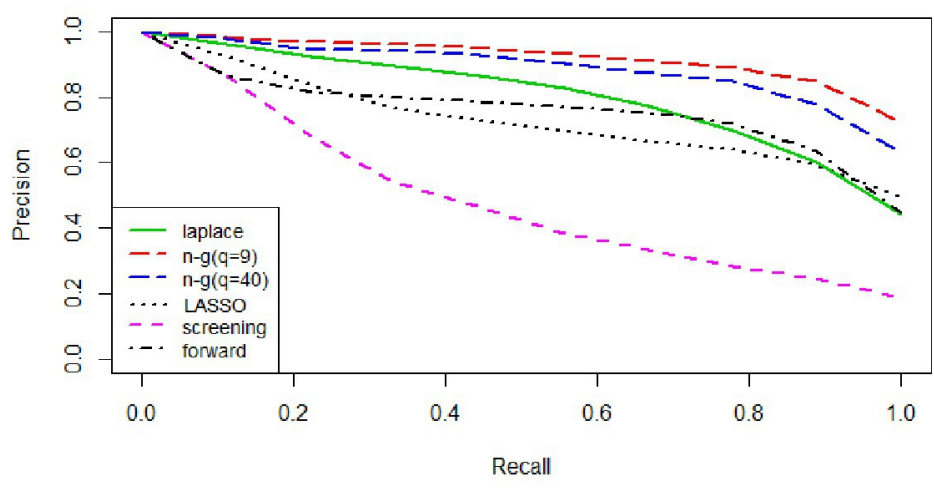

sam_11428_p1.eps

This article is protected by copyright. All rights reserved. 


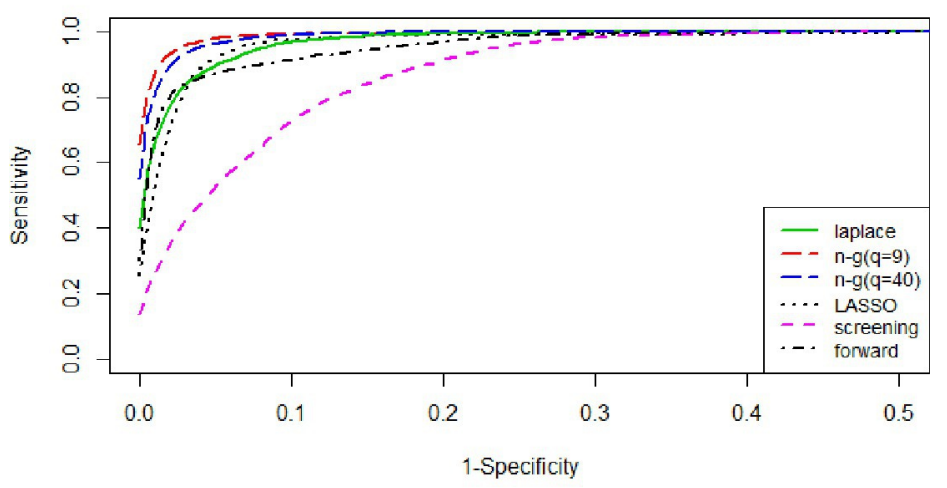

sam_11428_p2.eps

This article is protected by copyright. All rights reserved. 


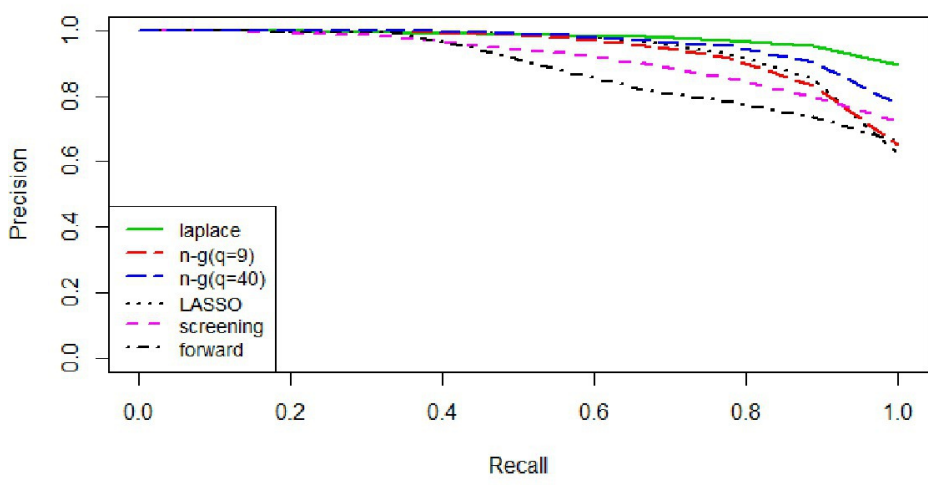

sam_11428_p3.eps

This article is protected by copyright. All rights reserved. 


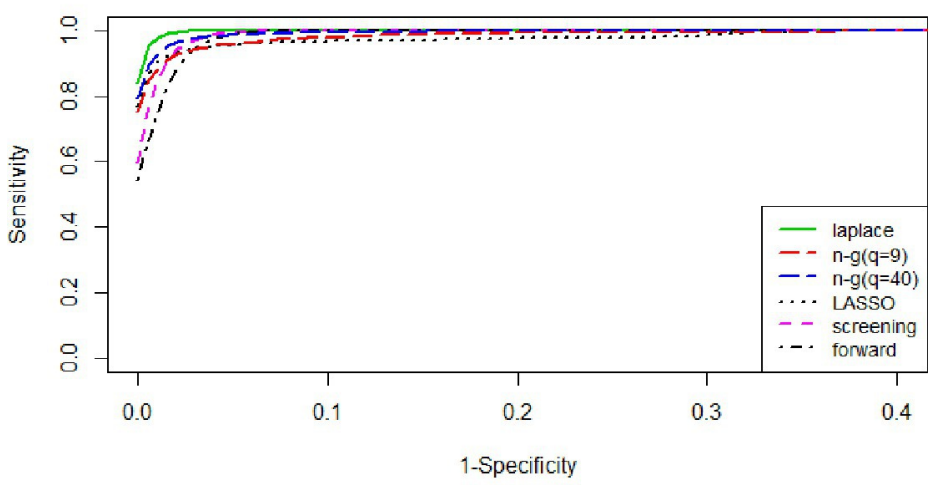

sam_11428_p4.eps

This article is protected by copyright. All rights reserved. 


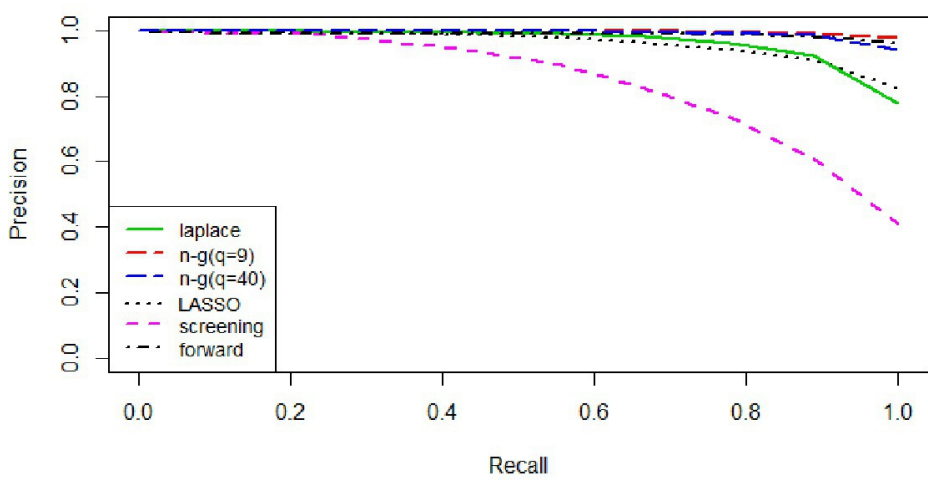

sam_11428_p5.eps

This article is protected by copyright. All rights reserved. 


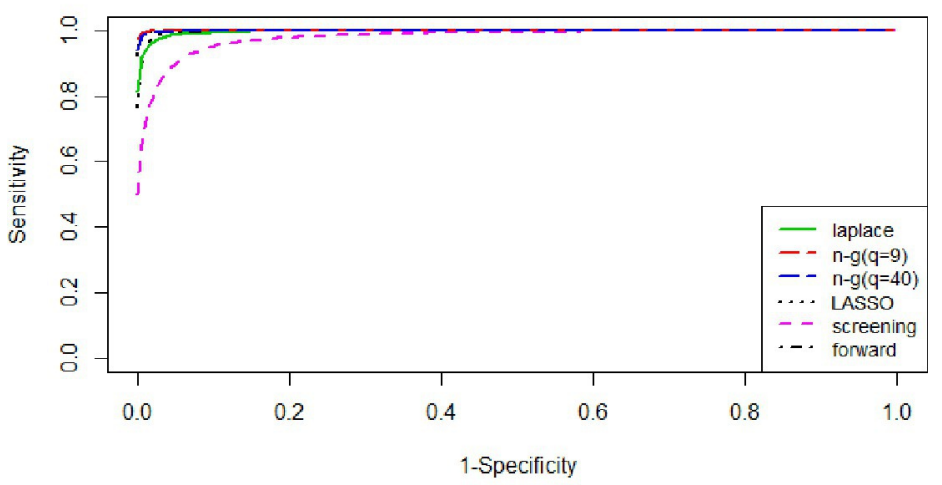

sam_11428_p6.eps

This article is protected by copyright. All rights reserved. 


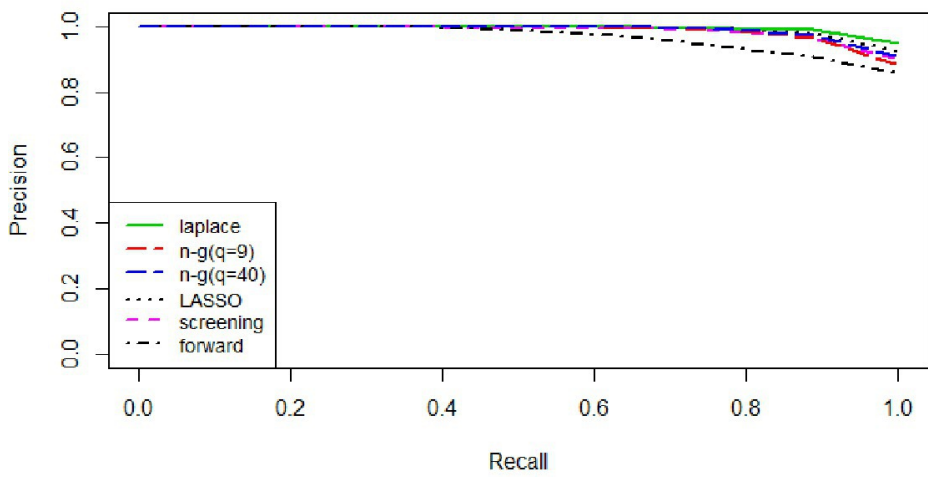

sam_11428_p7.eps

This article is protected by copyright. All rights reserved. 


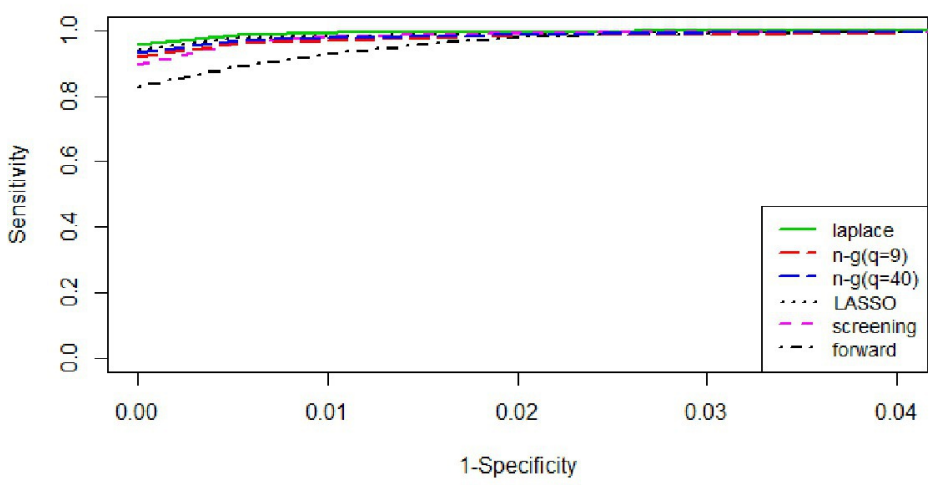

sam_11428_p8.eps

This article is protected by copyright. All rights reserved. 


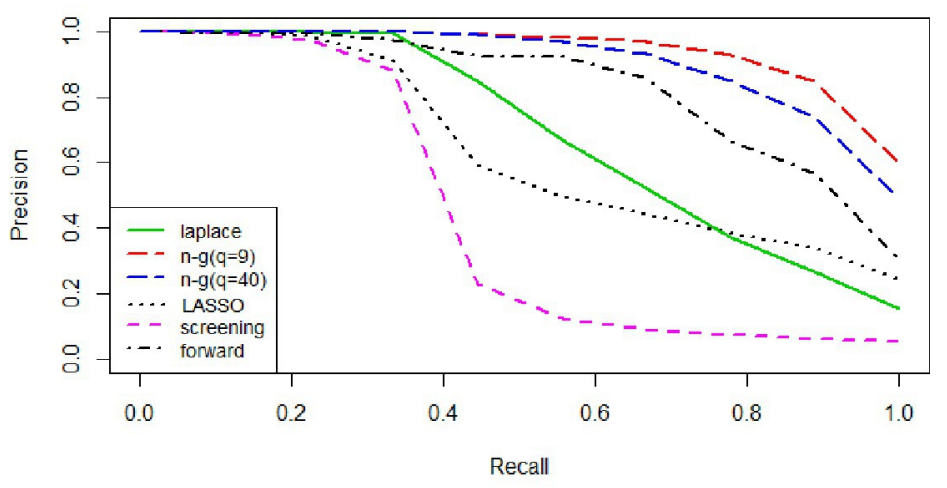

sam_11428_r1.eps

This article is protected by copyright. All rights reserved. 


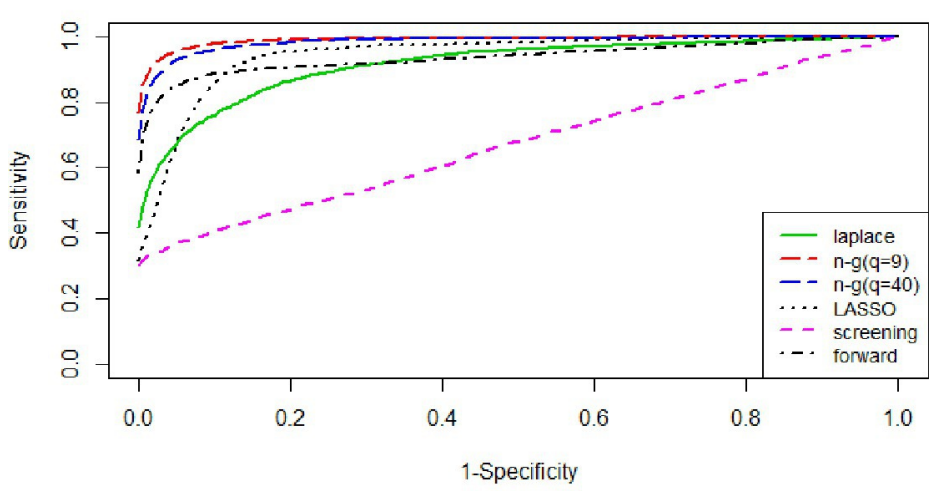

sam_11428_r2.eps

This article is protected by copyright. All rights reserved. 


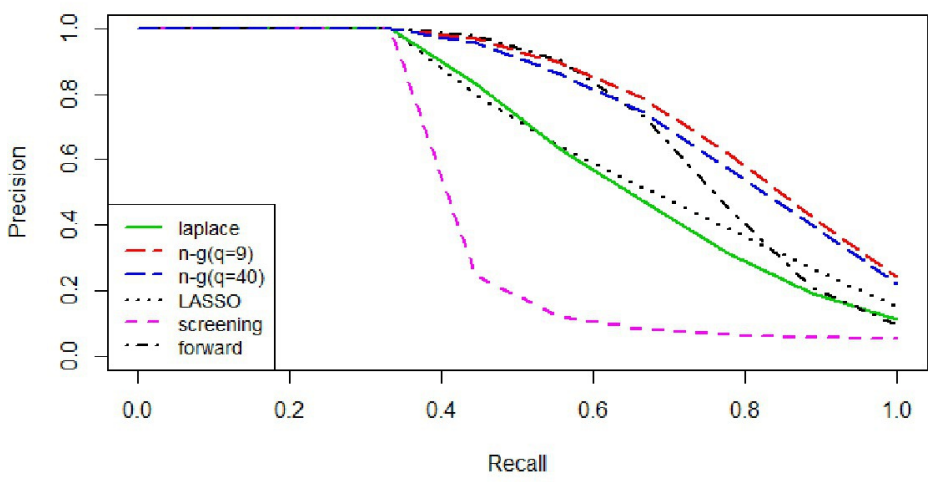

sam_11428_r3.eps

This article is protected by copyright. All rights reserved. 


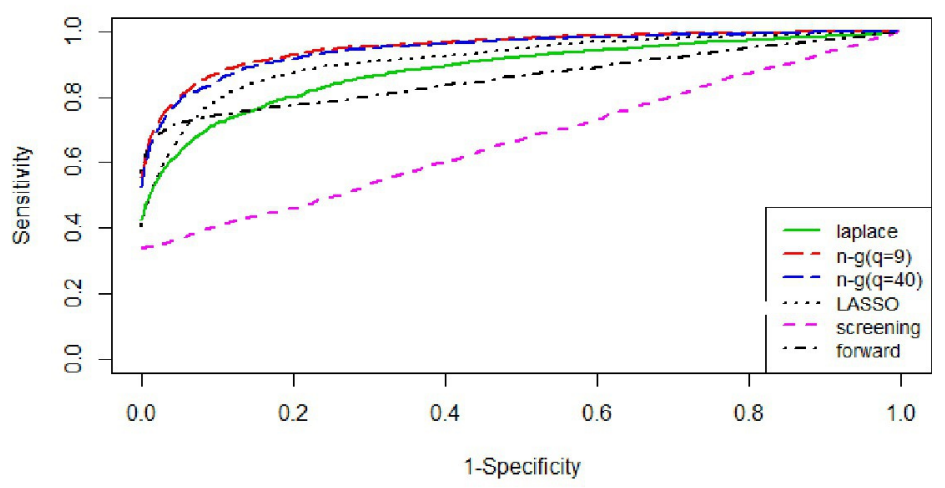

sam_11428_r4.eps

This article is protected by copyright. All rights reserved. 


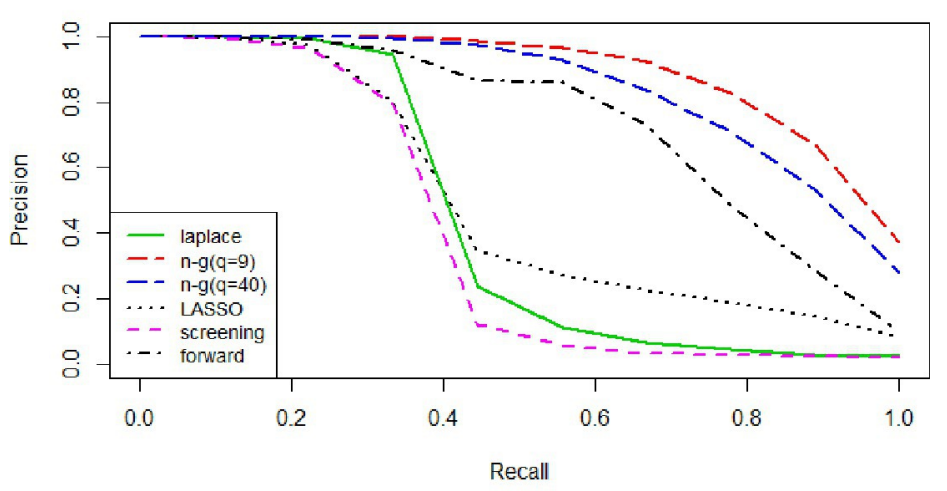

sam_11428_r5.eps

This article is protected by copyright. All rights reserved. 


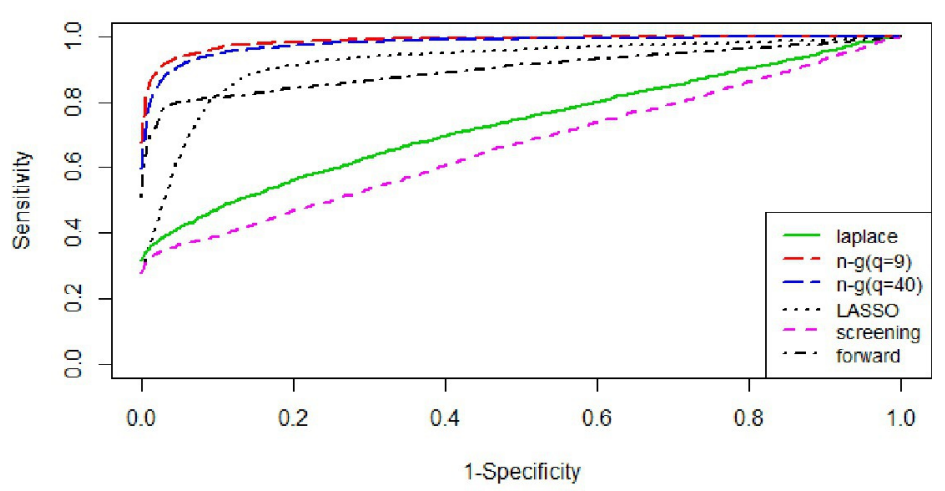

sam_11428_r6.eps

This article is protected by copyright. All rights reserved. 


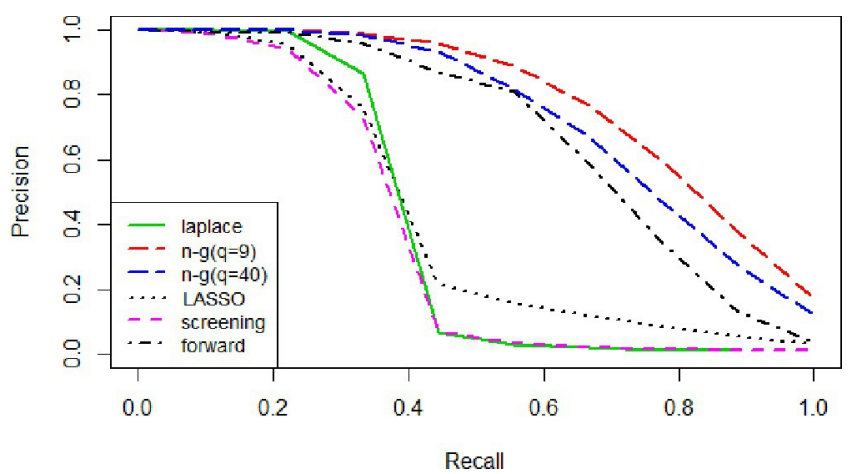

sam_11428_r9.eps

This article is protected by copyright. All rights reserved. 


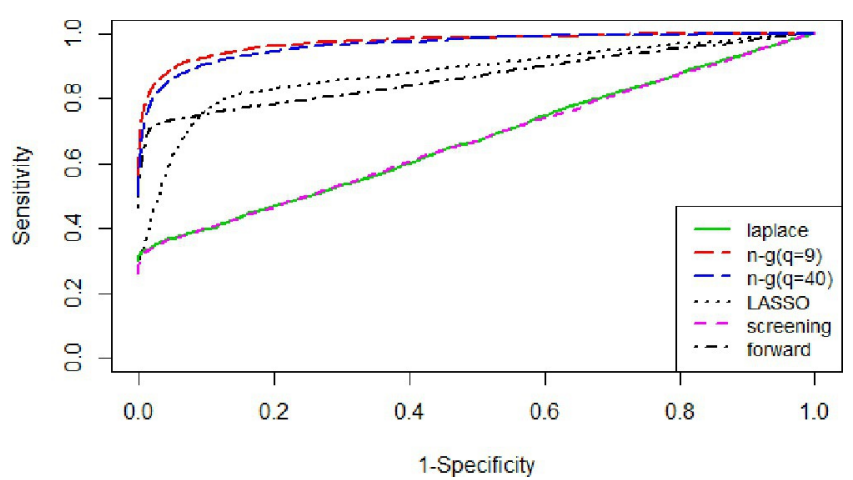

sam_11428_r10.eps

This article is protected by copyright. All rights reserved. 


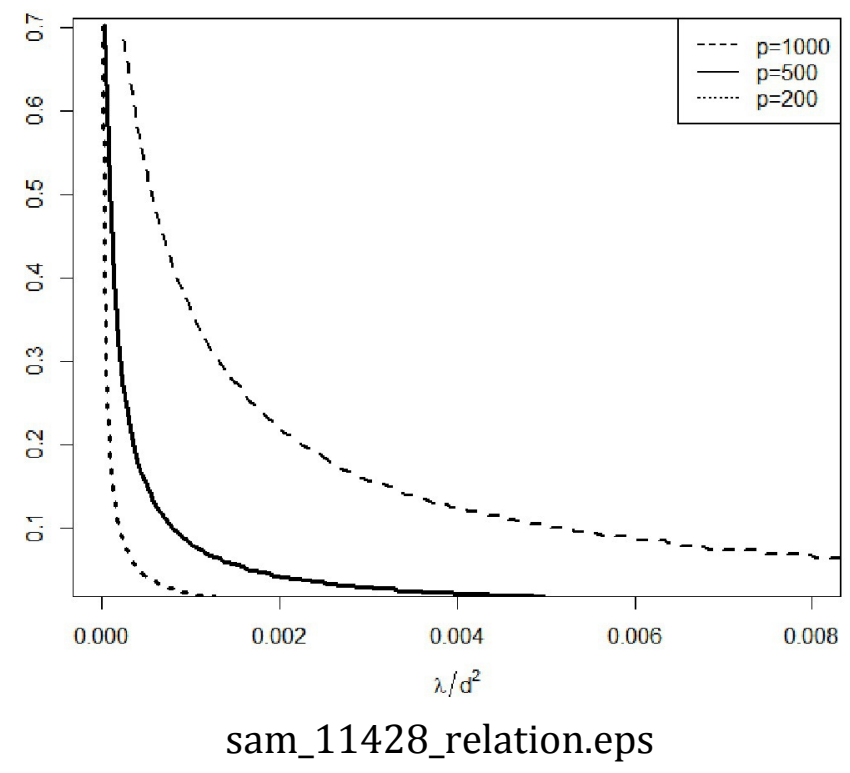

This article is protected by copyright. All rights reserved. 
Sparse Signal under Laplace Prior

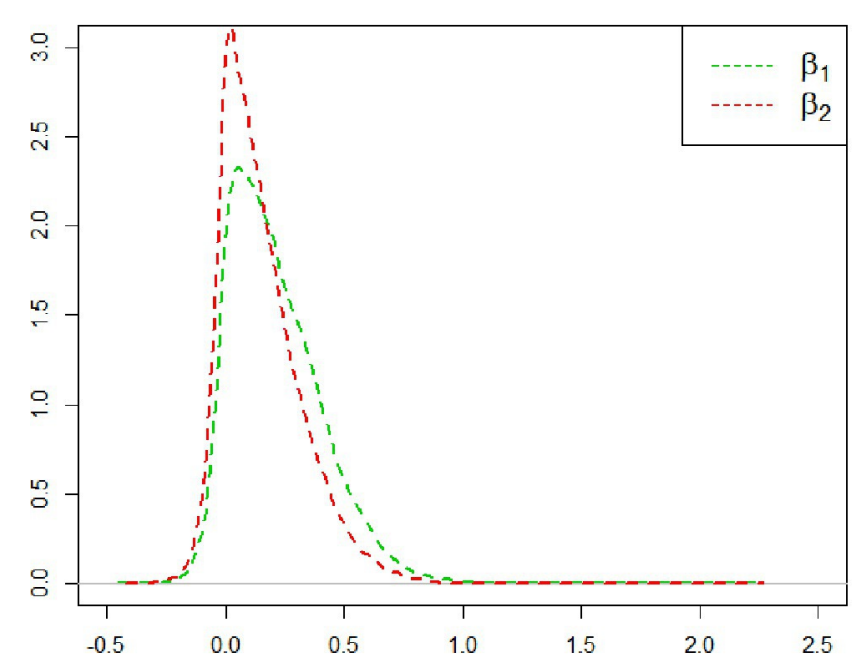

sam_11428_s_lp.eps

This article is protected by copyright. All rights reserved. 
Sparse Signal under Normal-Gamma Prior

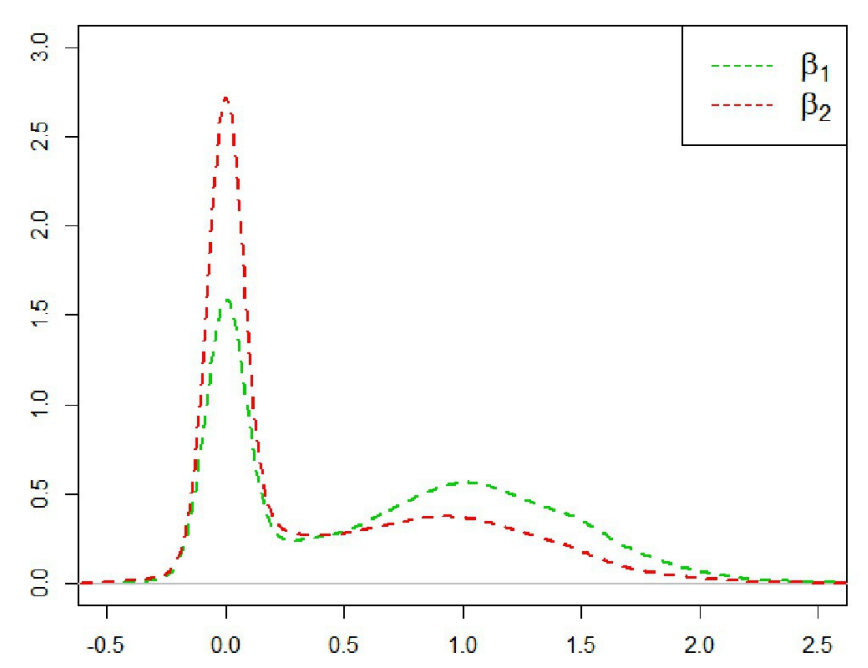

sam_11428_s_ng.eps

This article is protected by copyright. All rights reserved. 


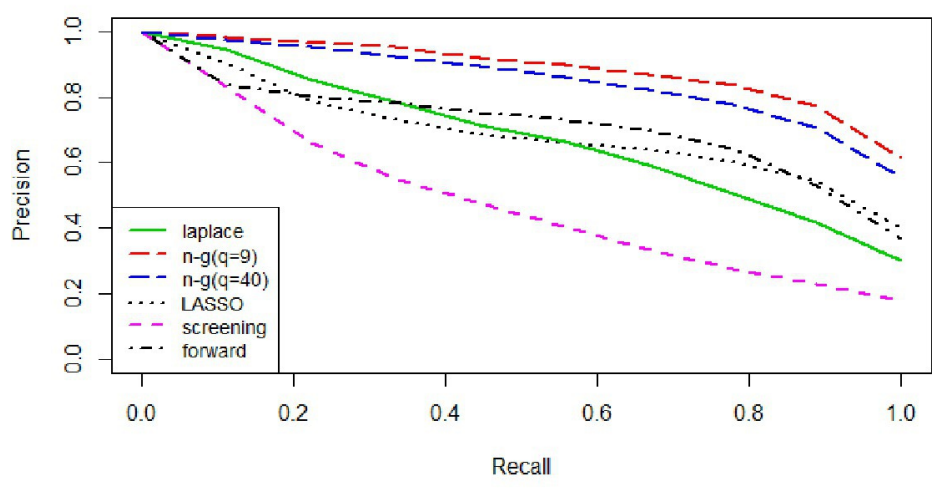

sam_11428_t1.eps

This article is protected by copyright. All rights reserved. 


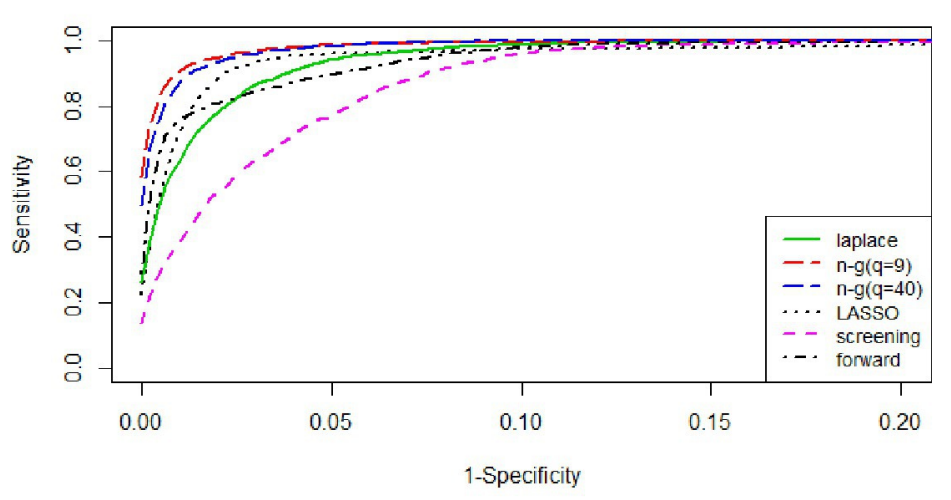

sam_11428_t2.eps

This article is protected by copyright. All rights reserved. 


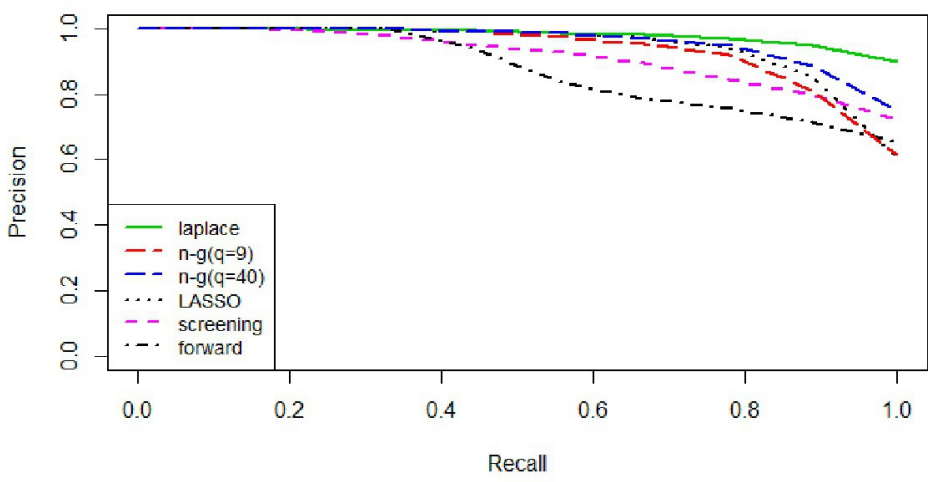

sam_11428_t3.eps

This article is protected by copyright. All rights reserved. 


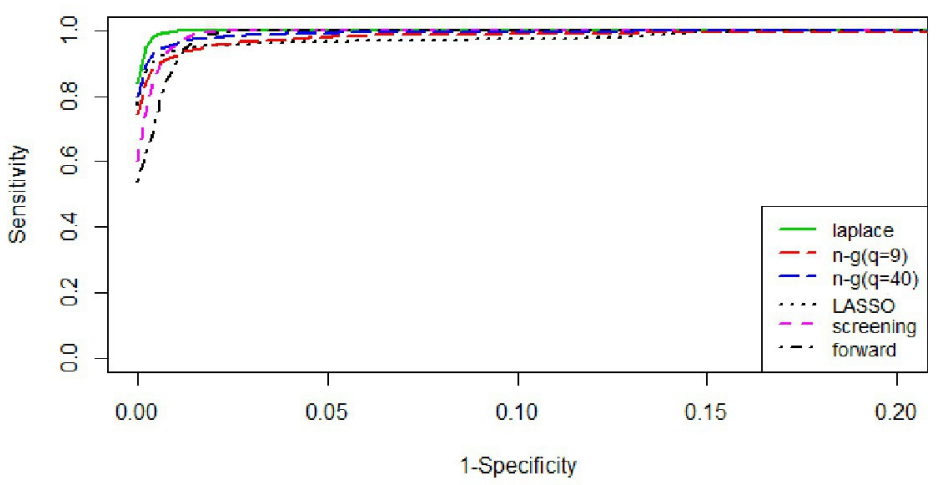

sam_11428_t4.eps

This article is protected by copyright. All rights reserved. 


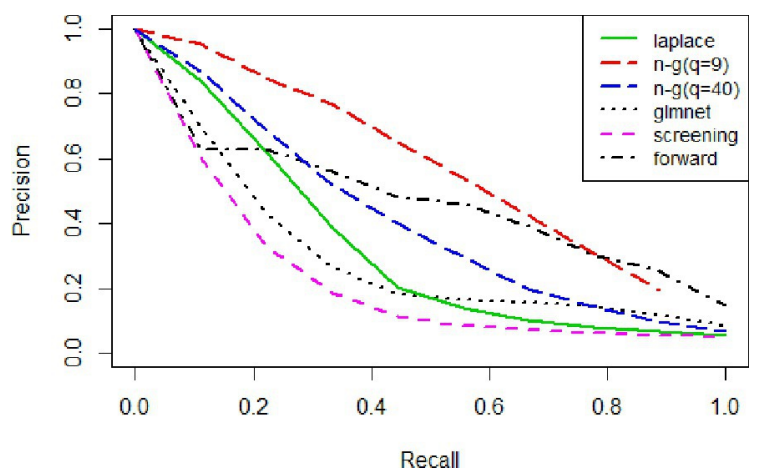

sam_11428_u1.eps

This article is protected by copyright. All rights reserved. 


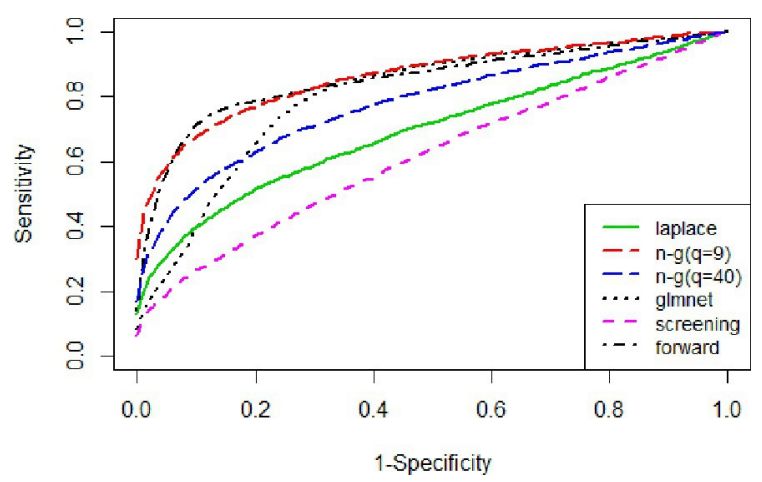

sam_11428_u2.eps

This article is protected by copyright. All rights reserved. 


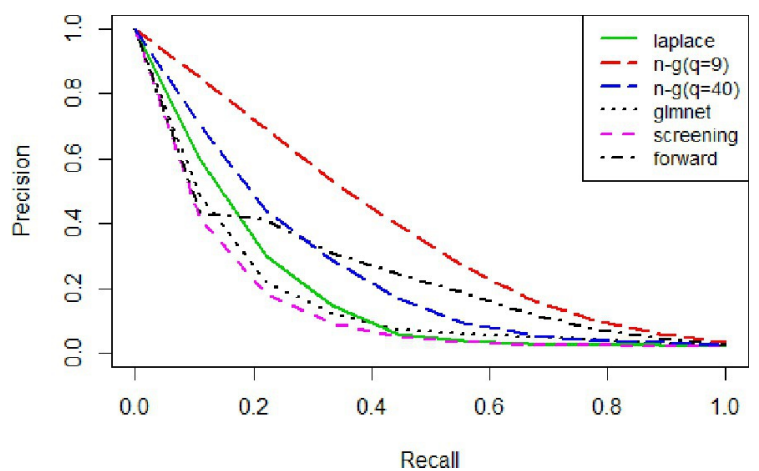

sam_11428_u3.eps

This article is protected by copyright. All rights reserved. 


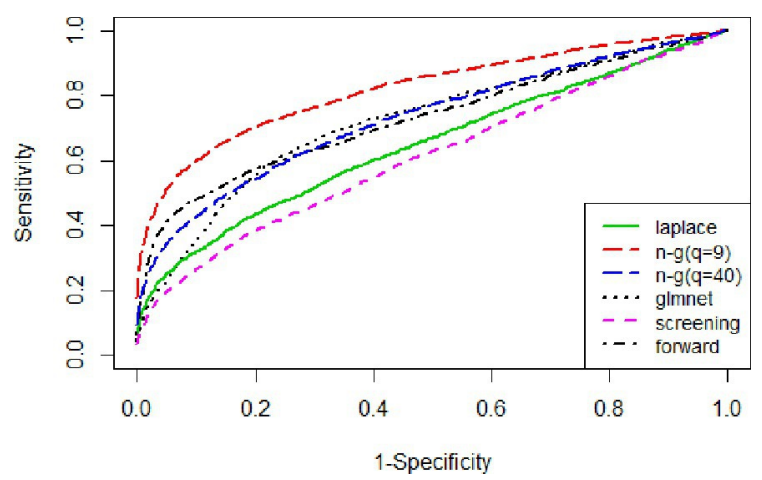

sam_11428_u4.eps

This article is protected by copyright. All rights reserved. 
Relationship of Important Proportion With Prior Variance

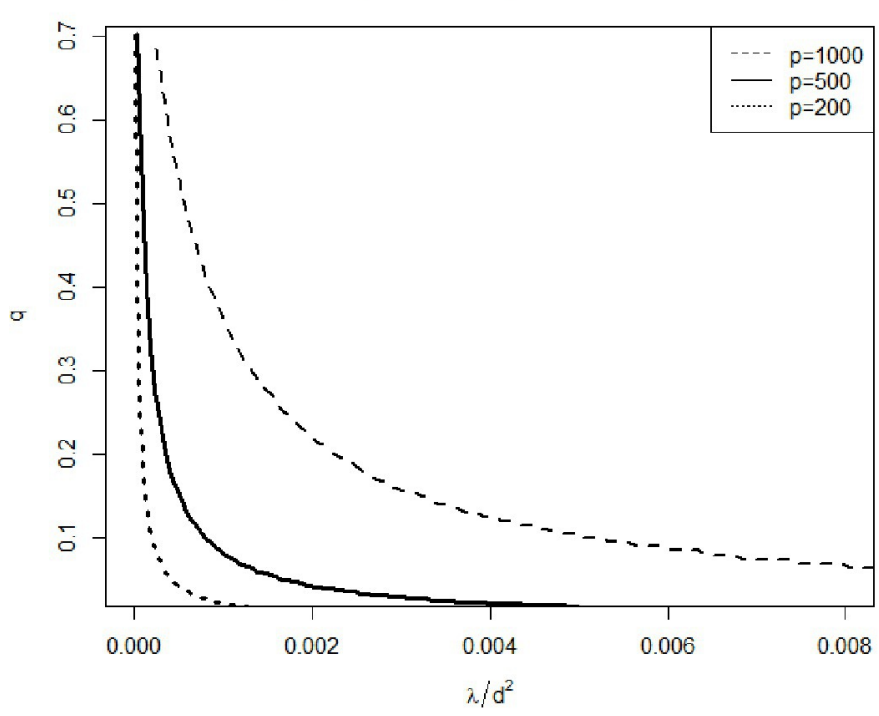

sam_11428_variance.eps

This article is protected by copyright. All rights reserved. 


\section{University Library}

\section{- M M I E R R V A gateway to Melbourne's research publications}

Minerva Access is the Institutional Repository of The University of Melbourne

Author/s:

Tian, Y;Bondell, HD;Wilson, A

Title:

Bayesian variable selection for logistic regression

Date:

2019-10-01

Citation:

Tian, Y., Bondell, H. D. \& Wilson, A. (2019). Bayesian variable selection for logistic regression. STATISTICAL ANALYSIS AND DATA MINING, 12 (5), pp.378-393. https:// doi.org/10.1002/sam.11428.

Persistent Link:

http://hdl.handle.net/11343/286050 